\title{
An early dynamical instability among the Solar System's giant planets triggered by the gas disk's dispersal
}

\section{Beibei Liu ( $\square$ bbliu@zju.edu.cn )}

Zhejiang Institute of Modern Physics \& Zhejiang University-Purple Mountain Observatory Joint Research Center for Astronomy

\section{Sean Raymond}

CNRS https://orcid.org/0000-0001-8974-0758

\section{Seth Jacobson}

Michigan State University https://orcid.org/0000-0002-4952-9007

Physical Sciences - Article

Keywords: astronomy, solar system, planets

Posted Date: December 30th, 2020

DOl: https://doi.org/10.21203/rs.3.rs-128128/v1

License: (c) (i) This work is licensed under a Creative Commons Attribution 4.0 International License. Read Full License 


\section{An early dynamical instability among the Solar System's giant planets triggered by the gas disk's dispersal}

Beibei Liu ${ }^{1,2 \star}$, Sean N. Raymond ${ }^{3}$, and Seth A. Jacobson ${ }^{4}$

${ }^{1}$ Zhejiang Institute of Modern Physics, Department of Physics \& Zhejiang University-Purple Mountain Observatory Joint Research Center for Astronomy, Zhejiang University, 38 Zheda Road, Hangzhou 310027, China

${ }^{2}$ Department of Astronomy and Theoretical Physics, Lund Observatory, Box 43, SE-22100, Sweden

${ }^{3}$ Laboratoire dAstrophysique de Bordeaux, Univ. Bordeaux, CNRS, B18N, allée Geoffroy SaintHilaire, 33615 Pessac, France

${ }^{4}$ Department of Earth and Environmental Sciences, Michigan State University, East Lansing, MI 48824, USA

*To whom correspondence should be addressed; Email: bbliu@zju.edu.cn

The Solar System's orbital structure is thought to have been sculpted by a dynamical instability among the giant planets ${ }^{1+4}$. Yet the instability trigger and exact timing have proved hard to pin down ${ }^{5+9}$. The giant planets formed within a gas-dominated disk around the young Sun. Motivated by giant exoplanet systems found in mean motion resonance ${ }^{10}$, hydrodynamical modeling has shown that while the disk was present the giant planets migrated into a compact orbital configuration, in a chain of resonances ${ }^{2.11}$. Here we use a suite of dynamical simulations to show that the giant planets' instability was likely triggered by the dispersal of the Sun's gaseous disk. As the disk evaporated from the inside-out, its inner edge swept successively across and dynamically perturbed each planet's orbit in turn. Saturn and each ice giants' orbits were torqued strongly enough to migrate outward. As a given planet migrated outward with the disk's inner edge the orbital configuration of the exterior system was compressed, triggering dynamical instability. The final orbits of our simulated systems match those of Solar System for a viable range of astrophysical parameters. Our results demonstrate that the giant planet instability happened as the gaseous disk dissipated, constrained by astronomical observations to be a few to ten million years after the birth of the Solar System ${ }^{12}$. Late stage terrestrial planet formation would occur mostly after such an early 
giant planet instability 13,14 , thereby avoiding the possibility of de-stabilizing the terrestrial planets ${ }^{15}$ and naturally accounting for the small mass of Mars relative to Earth and the mass depletion of the main asteroid belt 16 .

The evolution of gaseous protoplanetary disks is jointly controlled by viscous-driven accretion and wind-driven mass-loss $\frac{17}{17}$. In the early phase, the disk predominantly undergoes viscous evolution, as gas accretes at a considerable rate onto the central star for a few Myr. Meanwhile, the disk gas is photo-evaporated at a low rate by UV and X-ray photons from the central star $\frac{18}{1}$. Once the disk's surface density drops sufficiently that the disk accretion rate $\dot{M}$ is lower than the photoevaporating mass-loss rate $\dot{M}_{\text {pho }}$, the disk begins to dissipate from the inside-out 19 . The disk is truncated at a characteristic radius $R_{\text {in }}$; the gas interior to $R_{\text {in }}$ rapidly viscously drains onto the star, and an inner hole is formed. At this point the main outer disk is exposed to direct stellar radiation. Gas-free inner cavities have been directly detected in several disks that appear to be in the act of dispersing 20 . As photo-evaporation continues, the inner cavity expands outward until the disk is gone. While disks may also be evaporated from the outside-in by high-energy photons in dense stellar environments ${ }^{21}$, inside-out dispersal appears to be an ubiquitous process in disk clearing $\frac{18}{1}$. This dispersal can be characterized by three physical parameters: the onset mass-loss rate when photoevaporation takes over $\dot{M}_{\text {pho }}$, the final gas disk dispersal timescale $\tau_{\mathrm{d}}$, and the inner cavity expansion rate $v_{\mathrm{r}}$.

A dispersing protoplanetary disk has a direct effect on the orbital evolution of embedded planets. Angular momentum transfer between the planets' orbits and the gas leads to orbital migration, the shrinking or expansion of the planets' orbital radii22. Meanwhile, drag forces act to damp their orbital eccentricities and inclinations ${ }^{23}$. As the disk starts to disperse and an inner cavity is formed, planet migration at the inner disk edge is different than for planets fully embedded within the disk 24 . Embedded planets undergo "two-sided" gravitational torques from both the interior and exterior parts of the disk. In contrast, planets at the disk's inner edge only interact with gas on the outside of their orbits. As a result of larger-amplitude Lindblad and corotation torques, a planet below the mass threshold for opening a gap stops migrating inward at the disk inner edge 2526 . If the disk inner edge is itself moving radially outward due to disk dispersal, then the planet may subsequently migrate outward along with the expanding inner edge (see Supplementary Information). This mechanism is termed "rebound", and was first applied in the context 
of the magnetospheric cavity on sub-AU scales to explain the architecture of close-in super-Earth planets 24 27. In contrast, a planet massive enough to open a gap in the disk migrates interior to the cavity and its orbits is not affected as the cavity expands.

Here we show that the inside-out dispersal of the Sun's gaseous disk likely triggered a dynamical instability in the giant planets' orbits. Such an instability has been invoked to explain a number of the Solar System's features such as the giant planets' orbits $\frac{13}{3}$ and those of Trans-Neptunian objects ${ }^{28}$, Jupiter's co-orbital Trojan asteroids ${ }^{29}$, and the giant planets' irregular satellites 30 . Previous dynamical studies have almost universally relied on interactions between the giant planets and an outer planetesimal disk to trigger the instability on timescales of tens to hundreds of Myr $\frac{579}{31}$. In the framework of the disk dispersal-driven instability, the primordial outer planetesimal disk - whose existence is inferred from the present-day population of TransNeptunian objects ${ }^{\sqrt{22}}$ - did not trigger the instability but still contributed to shaping the system's orbital architecture.

Figure 1 presents a dynamical simulation of an instability triggered by the disk's dispersal. This simulation starts from a system with five giant planets, including Jupiter and Saturn and three ice giant planets, one of which was removed during the instability 33 . The planets' initial orbits reflect an earlier phase of growth and migration, such that each pair of neighboring planets is in mean motion resonance: Jupiter and Saturn are in the 2:1 resonance and all other planet pairs are in 3:2 resonances. The system was integrated taking into account the mutual gravitational forces between the planets and the effect of the dispersing gaseous disk.

The expanding edge of the inner disk cavity does not affect all planets equally. Because Jupiter opens a deep gap around its horseshoe region and the corresponding corotation torque diminishes, the rebound is quenched (see Supplementary Information) and Jupiter simply enters the cavity as the inner disk edge sweeps by. When the disk edge approaches Saturn at $t=0.6 \mathrm{Myr}$, the one-sided torque is strong enough to expand its orbit outward. Jupiter and Saturn exit their resonant state, as Saturn migrates outward with the expanding cavity, pushing into the orbits of the outer planets. The eccentricities of the ice giants become excited by this dynamical compression. Saturn is left behind and enters the cavity at $\sim 9 \mathrm{AU}$ at $t=0.7 \mathrm{Myr}$. Meanwhile, the innermost (blue in Figure 1) ice giant planet becomes excited to the point that its orbit crosses Saturn's, and the 
two planets undergo a close gravitational encounter. This triggers a dynamical instability and the system becomes chaotic: the third ice giant is scattered outward, while the innermost ice giant is eventually ejected towards interstellar space at $t \approx 0.8$ Myr after a series of close encounters with Jupiter. After the disk's inner edge has swept past all of the planets, their final orbits are close to those of the present-day Solar System giant planets (Figure 1).

It is worth noting that our simulations neglected the outer disk of planetesimals. Such a disk played a central role in triggering the instability and shaping the planets' final orbits in previous work ${ }^{15 / 79,31,33}$, which invoked a mass of $\sim 20$ Earth masses within 30 AU. Within the context of a rebound-triggered instability, the giant planets' orbits are basically decoupled from the mass in an outer planetesimal disk. Gravitational interactions with a remnant planetesimal disk would modestly expand the orbital radii of Uranus and Neptune and decrease their eccentricities on a 10-100 Myr timescale.

A rebound-triggered instability is consistent with the Solar System's orbital architecture. To demonstrate this we carried out $1.4 \times 10^{4}$ dynamical simulations like the one from Figure 1 . We varied three different aspects of these simulations (see Table 1). First, we tested a wide range of plausible starting configurations for the giant planets' orbits, including varying the number of ice giants $(2,3$, or 4$)$ and their initial resonant states. Second, we used a Monte Carlo method to test the effects of the three physical parameters - the onset mass-loss rate $\dot{M}_{\text {pho }}$, the disk dispersal timescale $\tau_{\mathrm{d}}$, and the rate of expansion of the inner cavity $v_{\mathrm{r}}$ - across the full range of astronomically-allowed values. Third, we ran each simulation twice; once including the effect of inside-out disk dissipation (with rebound) and once assuming the disk to dissipate smoothly at all radii (without rebound).

It is an ongoing challenge to use numerical simulations of the instability to concurrently match all of the Solar System's dynamical constraints 1/3/4/16/33/34 . Our goal is to demonstrate our new instability trigger, so we used two broad dynamical indicators to show that our simulated systems are indeed consistent with the global properties of the Solar System without attempting to match each detailed constraint. The first is the normalized angular momentum deficit ${ }^{35}$ : $\mathrm{AMD}=\sum m_{\mathrm{j}} \sqrt{a_{\mathrm{j}}}\left(1-\cos \left(i_{\mathrm{j}}\right) \sqrt{1-e_{\mathrm{j}}^{2}}\right) / \sum m_{\mathrm{j}} \sqrt{a_{\mathrm{j}}}$, where $m_{\mathrm{j}}, a_{\mathrm{j}}, e_{\mathrm{j}}$ and $i_{\mathrm{j}}$ are the mass, semimajor axis, eccentricity, and inclination of each giant planet. The AMD increases with increasing orbital eccentricities and inclinations, because eccentric or inclined orbits have a lower angular 
momentum than circular, coplanar ones at the same semimajor axes. The second indicator is the radial mass concentration ${ }^{36}: \mathrm{RMC}=\max \left(\sum m_{j} / \sum m_{j}\left[\log _{10}\left(a / a_{j}\right)\right]^{2}\right)$, which measures the degree of radial mass concentration in a given system, with higher RMC corresponding to a more tightly packed system.

Figure 2 shows the distribution of AMD and RMC values among a subset of our surviving planetary systems at the end of our simulations $(t=10 \mathrm{Myr})$. Distinctive features can be seen in Figure 2 when comparing the cases with and without rebound. First of all, dynamical instabilities are much less frequent when rebound is excluded. Among systems starting in 3:2 resonances (top panels), roughly $40 \%$ were unstable when rebound was ignored but more than $90 \%$ were unstable when it was included. In systems with a chain of 2:1 (Jupiter and Saturn) and 3:2 resonances (bottom panels), about 30\% were unstable when the rebound effect was ignored ${ }^{34}$ whereas roughly $80 \%$ went unstable when rebound was included. In addition, when rebound is taken into account, the distribution of final systems is more shifted from the initial low AMD and high RMC states towards final high AMD and low RMC states. A significant fraction of systems end up with an architecture similar to the Solar System (red star in Figure 2) with an instability timescale shorter than 10 Myr. This is not observed when rebound is not considered.

A rebound-triggered instability at the time of disk dispersal fills an important gap in Solar System chronology. Observations of the frequency of disks in star clusters of different ages find that the typical disk lifetime is a few to ten Myl ${ }^{12}$. Our Solar System's disk is thought to have lasted perhaps $5 \mathrm{Myr}$, spanning the age of the youngest chondritic meteorites 37 . The instability among the Solar System's giant planets was initially invoked as a delayed event to coincide with the "late heavy bombardment" 31 . However, recent re-appraisal of the cosmochemical constraints indicates that there was likely no late heavy bombardment ${ }^{38,39}$. Instead, constraints from a binary Jupiter Trojan ${ }^{6}$ and from ages of meteoritic inclusions ${ }^{8}$ suggest that the instability took place no later than 20-100 Myr after CAIs. An instability within 10 Myr after CAIs would have perturbed the final assembly of the terrestrial planets and may explain a number of features of the inner Solar System including the large Earth/Mars mass ratio and the excitation level of the asteroid belt 16 . In addition, a disk-driven instability removes the dynamical link between the giant planets' instability and the mass of the outer planetesimal disk, which may have important consequences for studies of planetesimal formation and cratering in the outer Solar System. For instance, the cratering record 
on outer Solar System moons does not appear to match predictions from models of the collisional evolution of the massive primordial Kuiper Belt needed to trigger the giant planets' instability 4041 .

The rebound-triggered instability occurs across all astrophysically-relevant disk parameter values. Figure 3 shows the distribution of outcomes of our simulations across the three parameters $\left(\dot{M}_{\text {pho }}, \tau_{\mathrm{d}}\right.$, and $v_{\mathrm{r}}$ ) for one set of starting conditions, with five giant planets in a chain of $2: 1$ and 3:2 resonances (run_C5R in Table 1). For this setup the systems with five surviving planets (black edgecolor) are likely to form when the onset mass-loss rate $\dot{M}_{\text {pho }}$ is low. We note that for the above explored range of parameters, the final architectures are not strongly sensitive to the disk dispersal timescale $\tau_{\mathrm{d}}$ and cavity expansion rate $v_{\mathrm{r}}$. Solar System analogs (magenta edgecolor) preferentially form when $\dot{M}_{\text {pho }} \lesssim 10^{-9} \mathrm{M}_{\odot} \mathrm{yr}^{-1}$. Such ranges of parameters are also broadly consistent with astrophysical constraints. See the Supplementary Information for equivalent plots for our other starting conditions and for a discussion of observational constraints on the relevant parameters.

Our results show that the dispersal of the nebular gas may act as an instability trigger for nascent planetary systems. Specifically, the rebound effect may explain the apparent ubiquity of dynamical instability observed in exoplanetary systems ${ }^{42}$. The broad eccentricity distribution of giant exoplanets can be matched if $75-95 \%$ of all giant planet systems we see are the survivors of dynamical instabilities ${ }^{43}$. Likewise, the orbital period ratio distribution of close-in small planets is consistent with the vast majority of systems having undergone dynamical instabilities ${ }^{44}$. Thus, the rebound effect during disk dispersal may be a near universal process effecting not just our Solar System but planetary systems across the galaxy.

Correspondence and requests for materials Correspondence and requests for materials should be addressed to B. L. (email: bbliu@zju.edu.cn).

Acknowledgements . B. L. is supported by the European Research Council (ERC Consolidator Grant 724687- PLANETESYS), the Swedish Walter Gyllenberg Foundation, and start-up grant of Bairen program from Zhejiang University. S. N. R. is grateful to the CNRS's PNP program for funding.

Author contributions S. N. R. and S. A. J. proposed this idea and initiated the collaboration. B. L. examined the feasibility and conducted numerical simulations. All authors contributed to analyzing and discussing the numerical results, editing and revising the manuscript. 
Author information The authors declare that they have no competing interests. 


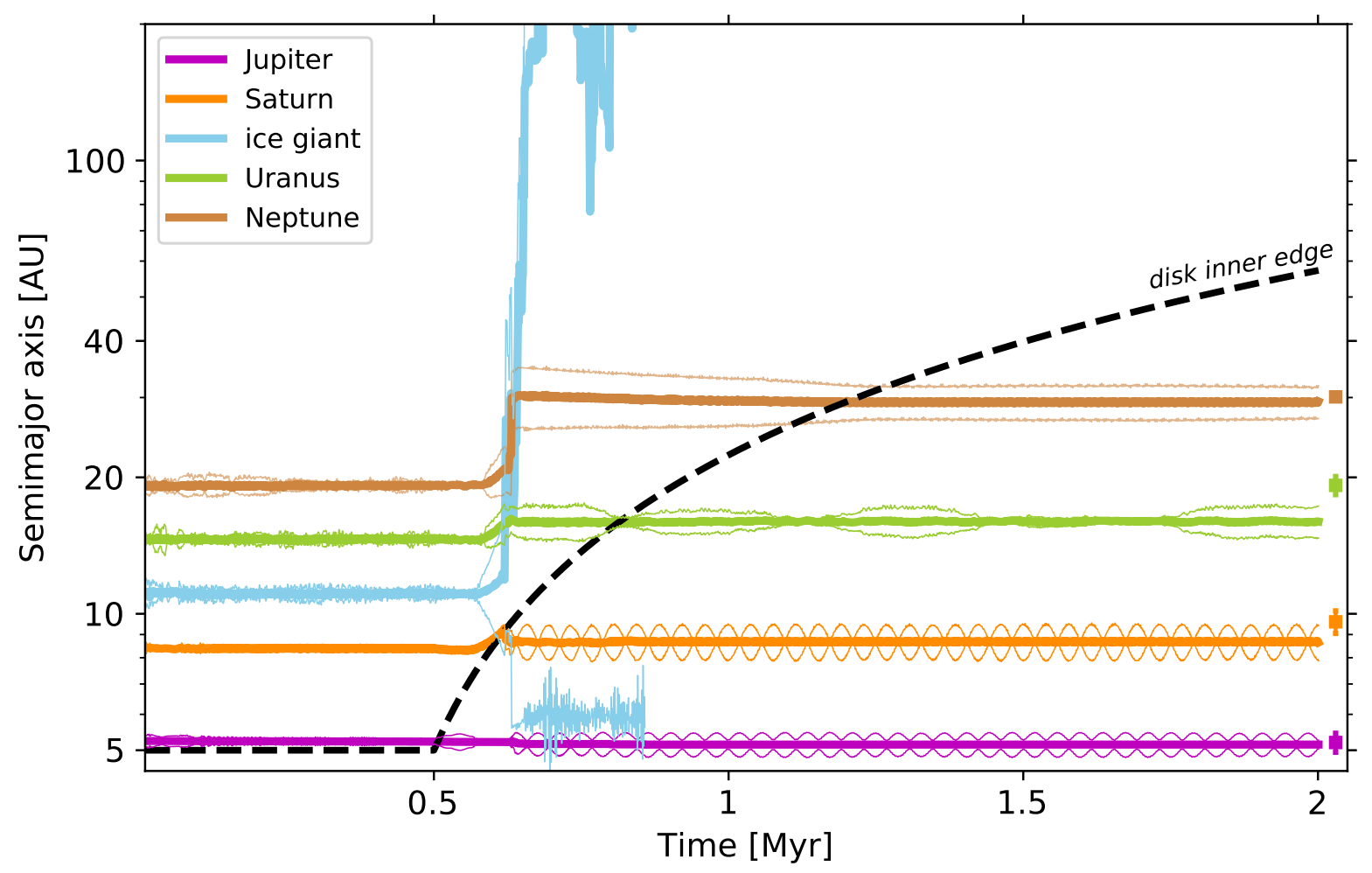

Figure 1: An early dynamical instability triggered by the dispersal of the Sun's protoplanetary disk. The initial system consisted of five giant planets: Jupiter (magenta), Saturn (orange), and three ice giants, each of which is $15 M_{\oplus}$ and one of which (blue) was ejected into interstellar space during the instability. The curves show the orbital evolution of each body including its perihelion, semimajor axis, and aphelion (thin, thick, and thin curves, respectively). The black dashed line tracks the edge of the disk's expanding inner cavity. We do not follow the early evolution through the entire gas-rich disk phase, so the onset of disk dispersal is set arbitrarily to be $0.5 \mathrm{Myr}$ after the start the simulation. The semimajor axes and eccentricities of the present-day giant planets are shown at the right, with vertical lines extending from perihelion to aphelion. The adopted disk parameters are: $\dot{M}_{\text {pho }}=4 \times 10^{-10} \mathrm{M}_{\odot} \mathrm{yr}^{-1}, \tau_{\mathrm{d}}=8.6 \times 10^{5} \mathrm{yr}$, and $v_{\mathrm{r}}=35 \mathrm{AU} \mathrm{Myr}^{-1}$. 


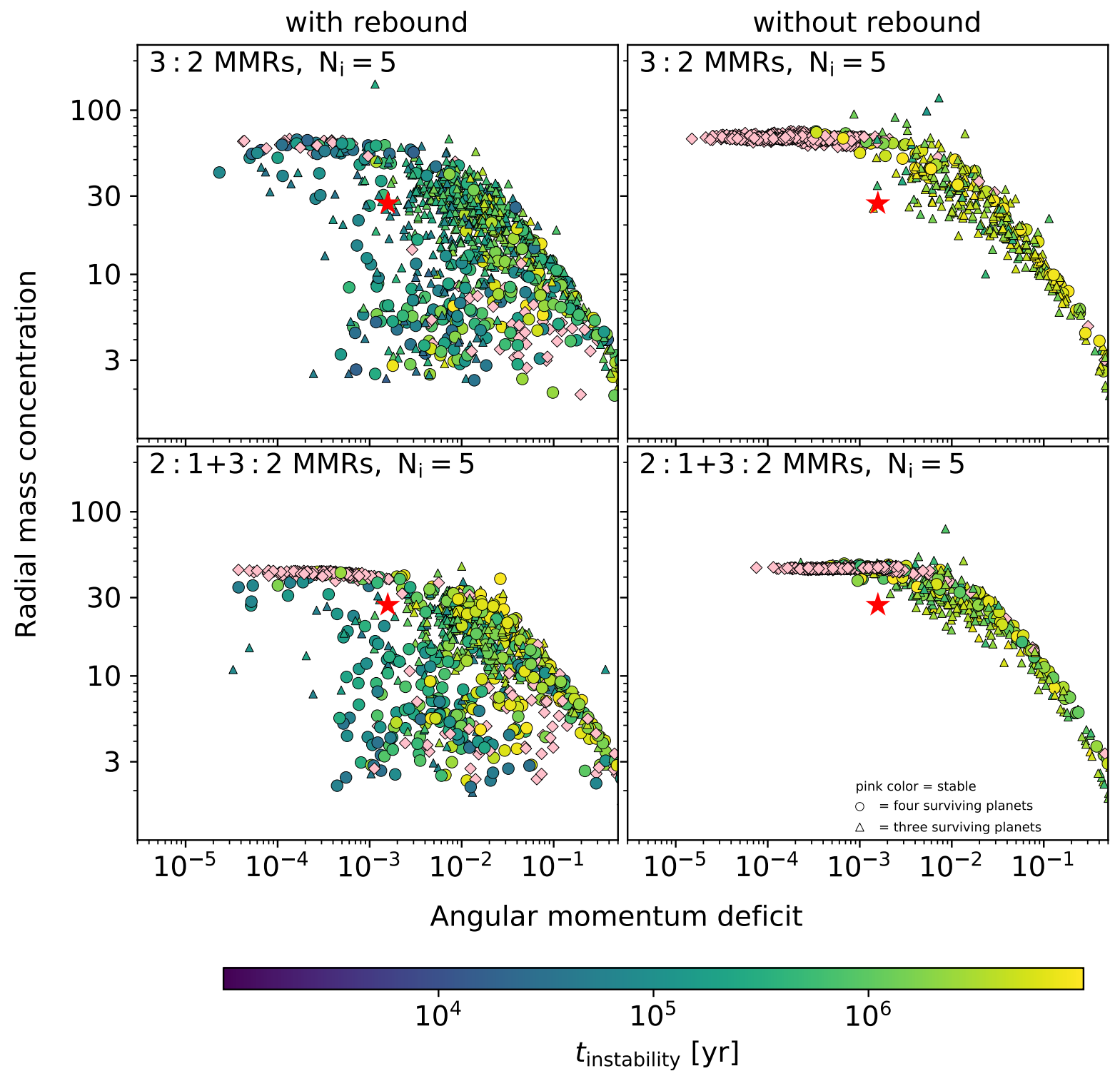

Figure 2: The radial mass concentration (RMC) and normalized angular momentum deficit (AMD) distributions among surviving planetary systems in a subsample of our simulations.

The simulations on the left included the rebound effect and those on the right did not. Each simulation started with our four present-day giant planets plus one additional ice giant planet. In the top panels, the giant planets were initially placed in a chain of 3:2 orbital resonances. In the bottom panels, Jupiter and Saturn were in 2:1 resonance and other neighboring planets in 3:2 resonances. Each symbol represents the outcome of a given simulation. The color indicates the timing of the instability after the start of gas disk dispersal; pink systems did not undergo an instability (no collision/ejection). Circles and triangles correspond to systems with four and three or fewer surviving planets, respectively. The Solar System is marked as a red star for comparison. Comparable figures starting from different orbital configurations are included in the Supplementary Information. 

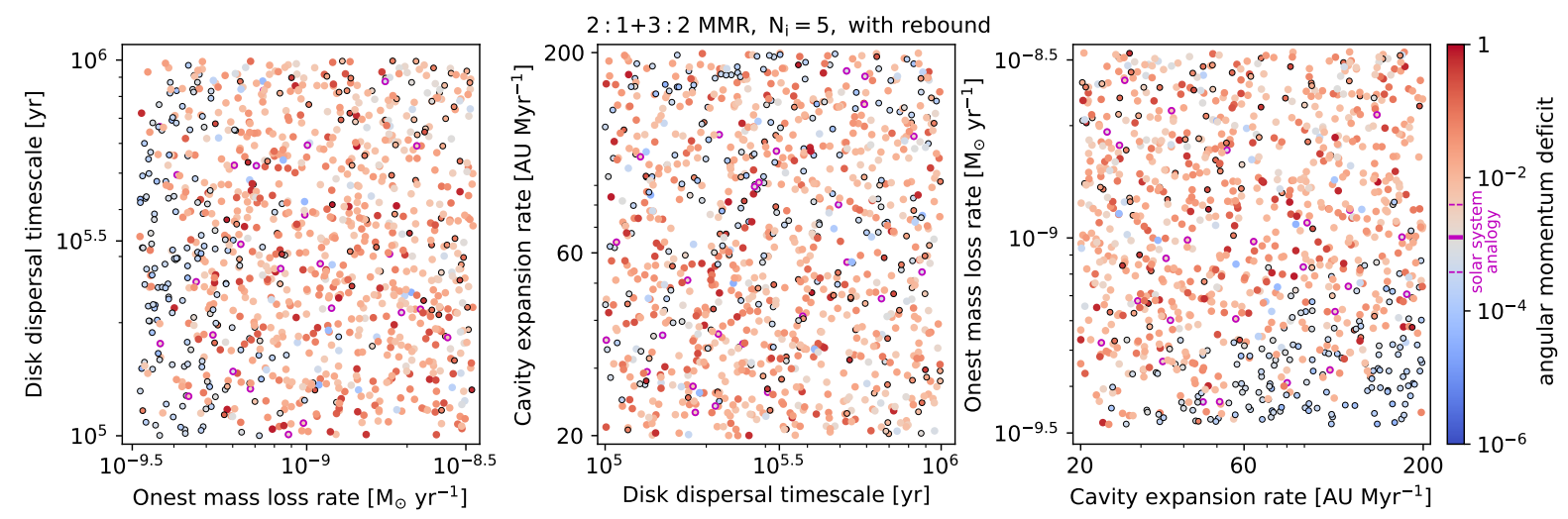

Figure 3: Simulation outcomes as a function of disk parameters. Each simulation started with four four present-day giant planets plus one ice giant planet in a series of $2: 1$ and 3:2 orbital resonances (run_C5R in Table 1). The color bar corresponds to the system's angular momentum deficit (AMD). The circles with black edgecolor refer to the systems whose planets all survive in the end, while the circles with magenta edgecolor represent the Solar System analogs, defined as systems with four surviving planets in the right order and their AMDs and RMCs are within a factor of three of our Solar System's values. Comparable figures starting from different orbital configurations are included in the Supplementary Information. 


\section{Methods}

Disk model. The evolution of protoplanetary disks is thought to be driven by a combination of internal stress from the gas' viscosity and external stellar UV/X-ray photoevaporation 17/18/45. At early times the influence of stellar photoevaporation is negligible and the disk evolves viscously in a quasi-steady state such that $\dot{M}=3 \pi \nu \Sigma$, where $\dot{M}$ is the gas disk accretion rate, $\Sigma$ is the gas surface density, $\nu=\alpha c_{\mathrm{S}} H$ is the gas turbulent viscosity ${ }^{46}, \alpha$ is the viscous efficiency parameter, and $c_{\mathrm{S}}$ and $H$ are the gas disk sound speed and scale height, respectively. At late times, after the disk accretion rate $\dot{M}$ drops below the stellar photoevaporation mass-loss rate $\dot{M}_{\text {pho, }}$, disk dissipation is dominated by the photoevaporative wind originated from stellar high-energy radiation 1947 .

When the thermal energy of disk gas is greater than its gravitational bounding energy beyond a threshold disk radius $R_{\text {in }}$, the disk gas escapes as a wind. Gas interior to $R_{\text {in }}$ is rapidly accreted through a short, viscous timescale. Hence, an inner disk cavity is opened and the main outer disk is optically thin to the direct radiation from the central star 19 . Under such a circumstance, the photoevaporating mass-loss rate is given by 18

$$
\dot{M}_{\text {pho }} \simeq 10^{-9}\left(\frac{\Phi}{10^{41} \mathrm{~s}^{-1}}\right)^{1 / 2}\left(\frac{R_{\text {in }}}{5 \mathrm{AU}}\right)^{1 / 2} \mathrm{M}_{\odot} \mathrm{yr}^{-1},
$$

where $\Phi$ represents the ionizing flux from the central star and $R_{\text {in }}$ is the initial size of inner cavity. We note that photoevaporation is a complicated process coupled with radiative transfer and disk hydrodynamics. The mass-loss rate depends on the primary incident spectrum of the central star (EUV, FUV, or X-ray), grain abundance, and disk chemistry. The fiducial value of the onset massloss rate $\dot{M}_{\mathrm{pho}}$ of $10^{-9} \mathrm{M}_{\odot} \mathrm{yr}^{-1}$ in Eq. $[1]$ is taken from models of EUV-driven stellar radiation 19. If the photoevaporation is instead dominated by $\mathrm{X}$-ray radiation, the mass-loss rate can be even higher ${ }^{47}$. We vary $\dot{M}_{\text {pho }}$ from $3 \times 10^{-9}$ to $3 \times 10^{-10} \mathrm{M}_{\odot} \mathrm{yr}^{-1}$ in the parameter study.

In this photoevaporation-driven disk dispersal phase, the gas surface density can be written

$$
\Sigma(t)=\Sigma_{\text {pho }} \exp \left[-\frac{t}{\tau_{\mathrm{d}}}\right]=\frac{\dot{M}_{\mathrm{pho}}}{3 \pi \nu} \exp \left[-\frac{t}{\tau_{\mathrm{d}}}\right],
$$

where $\Sigma_{\text {pho }}$ is the gas surface density when the stellar photoevaportation becomes dominant and $\tau_{\mathrm{d}}$ is the disk dispersal timescale. Noticeably, two different timescales are related to the gas disk dissipation. First, the disk lifetime is inferred to be $1-10 \mathrm{Myr}$ with a median value of $\sim 3 \mathrm{Myr} 4849$. 
Second, since a small fraction of young stars have evolved disks being a transition between diskbearing and diskless, the disk clearing is expect to be very rapid, typically an order of magnitude shorter than the disk lifetime ${ }^{50,52}$. Based on these observational constraints, it is reasonable to assume that the disk dispersal timescale $\tau_{\mathrm{d}}$ is in a range between 0.1 and $1 \mathrm{Myr}$.

In this paper we assume a optically thin stellar irradiation dominant flaring disk ${ }^{53}$ in which the disk temperature and aspect ratio can be expressed as

$$
T=\left(\frac{L_{\star}}{16 \pi \sigma_{\mathrm{SB}} r^{2}}\right)^{1 / 4}=T_{\mathrm{in}}\left(\frac{r}{R_{\mathrm{in}}}\right)^{-1 / 2}, h=\frac{H}{r}=\sqrt{\frac{k_{\mathrm{B}} T / \mu m_{\mathrm{p}}}{G M_{\star} / r}}=h_{\mathrm{in}}\left(\frac{r}{R_{\mathrm{in}}}\right)^{1 / 4}
$$

where $M_{\star}$ and $L_{\star}$ are the stellar mass and luminosity, $r$ is the disk radial distance, $G$ is the gravitational constant, $\sigma_{\mathrm{SB}}$ is the Stefan-Boltzmann constant, $k_{\mathrm{B}}$ is the Boltzmann constant, $\mu$ is the gas mean molecule weight and $m_{\mathrm{p}}$ is the proton mass. Ref ${ }^{53}$ calculated that $h=0.033$ at 1 AU by adopting $L_{\star}=1 L_{\odot}$. However, during the pre-main-sequence (age of $<$ a few Myr), the Sun underwent gravitational contraction and was far more luminous $\left(\sim 1-10 L_{\odot}\right.$, along the Hayashi track) than its main-sequence stage. Hence, we choose a higher luminosity for a solar-mass pre-mainsequence star of $L_{\star}=4 L_{\odot}$ and assume it remains constant during relatively rapid disk dispersal phase. In this circumstance, the disk aspect ratio is 0.04 at $1 \mathrm{AU}$. We adopt $R_{\mathrm{in}}=5 \mathrm{AU}$ and therefore $h_{\mathrm{in}}=0.06$ and $T_{\mathrm{in}}=180 \mathrm{~K}$, where the notation $X_{\mathrm{in}}$ indicates the quantity $X$ evaluated at the inner disk edge $R_{\text {in }}$ when the stellar photoevaporation dominates. Based on the quasi-steady state assumption, we rewrite the gas surface density as

$$
\Sigma(t)=\frac{\dot{M}_{\mathrm{pho}}}{3 \pi \nu} \exp \left[-\frac{t}{\tau_{\mathrm{d}}}\right]=\Sigma_{\mathrm{in}}\left(\frac{r}{R_{\mathrm{in}}}\right)^{-1} \exp \left[-\frac{t}{\tau_{\mathrm{d}}}\right]
$$

where $\Sigma_{\text {in }}=4 \mathrm{~g} \mathrm{~cm}^{-2}$ for the fiducial disk parameters of $\dot{M}_{\mathrm{pho}}=10^{-9} \mathrm{M}_{\odot} \mathrm{yr}^{-1}$ and $\alpha=0.005$.

As disk gas disperses from inside-out the inner cavity spreads outward. For simplicity, we adopt a constant $v_{\mathrm{r}}$ to represent the cavity expansion speed. This quantity is not well constrained from a observational perspective. The cavity expansion rate should be principally determined by stellar ionizing flux, size and mass of the disk, and hence, it is not a full-independent variable. Nonetheless, when the dispersal time and disk mass are fixed, the expansion rate directly reflects the size of the disk. To a first order approximation, for a disk with an outer edge $R_{\text {out }}$ of $30 \mathrm{AU}$ and dispersal timescale of $0.5 \mathrm{Myr}$, the cavity expansion rate approximates as 
$v_{\mathrm{r}} \sim R_{\text {out }} / \tau_{\mathrm{d}}=60 \mathrm{AU} \mathrm{Myr}^{-1}$. Due to the uncertainties in $R_{\text {out }}$ and $\tau_{\mathrm{d}}$, the cavity expansion speed could plausibly span two orders of magnitude from 10 to $10^{3} \mathrm{AU} \mathrm{Myr}^{-1}$.

In brief, the final photoevaportation-driven disk dispersal can be featured by three key model parameters: the disk dispersal timescale $\tau_{\mathrm{d}}$, the onset mass-loss rate when photoevaporation dominates $\dot{M}_{\text {pho }}$, and the inner disk cavity expansion speed $v_{\mathrm{r}}$.

Planet migration. We summarize the formulas of planet migration torques based on ref. 24 . When the planet is far from the inner edge of the disk, it feels the torque from disk gas on its both sides. The two-sided torque is adopted from Eq. (49) of ref. ${ }^{54}$, which reads

$$
\frac{\Gamma_{2 \mathrm{~s}}}{m_{\mathrm{p}}\left(r_{\mathrm{p}} \Omega_{\mathrm{p}}\right)^{2}}=-2.3 q_{\mathrm{d}} \frac{q_{\mathrm{p}}}{h^{2}},
$$

where $\Omega_{\mathrm{K}}$ is the Keplerian angular velocity, and $q_{\mathrm{d}} \equiv \Sigma_{\mathrm{g}} r_{\mathrm{p}}^{2} / M_{\star}$ and $q_{\mathrm{p}} \equiv m_{\mathrm{p}} / M_{\star}$ are the mass ratio between the local gas disk and star and mass ratio between the planet and star, respectively. When the planet is at the disk edge, only the one-sided torque exists, which is given by

$$
\frac{\Gamma_{1 \mathrm{~s}}}{m_{\mathrm{p}}\left(r_{\mathrm{p}} \Omega_{\mathrm{p}}\right)^{2}}=C_{\mathrm{hs}} q_{\mathrm{d}}\left(\frac{q_{\mathrm{p}}}{h^{3}}\right)^{1 / 2}+C_{\mathrm{L}} q_{\mathrm{d}} \frac{q_{\mathrm{p}}}{h^{3}},
$$

where the first and second terms on the right side of Eq. (6) are the corotation and Lindblad torque components, and $C_{\mathrm{hs}}=2.46, C_{\mathrm{L}}=-0.65$ are the corresponding coefficients. In order to derive the corotation torque in Eq. (6), we assume that at the disk inner edge, the gas removal time $t_{\text {removal }}$ is faster than the gas liberation time in the planet horseshoe region $t_{\text {lib }}$. We demonstrate that this is a justified treatment as follows. First, at the inner disk edge the gas removal time should be no longer than the viscous diffusion time $t_{\mathrm{vis}}$; otherwise the gas would be accumulated. Thus, we have $t_{\text {removal }} \lesssim t_{\mathrm{vis}} \simeq x_{\mathrm{hs}}^{2} / \nu$, where $x_{\mathrm{hs}} \sim r_{\mathrm{p}} \sqrt{q_{\mathrm{p}} / h}$ is the half-width of the planet horseshoe region. The gas liberation time for a planet can be written as $t_{\mathrm{lib}} \sim 8 \pi r /\left(3 \Omega_{\mathrm{K}} x_{\mathrm{hs}}\right)$. Then $t_{\text {removal }}<t_{\text {lib }}$ is required for one-sided corotation torque in Eq. (6). The above condition is satisfied as long as $t_{\mathrm{vis}}<t_{\mathrm{lib}}$, and one can obtain that $q \lesssim 8 \pi / 3 \alpha^{2 / 3} h^{7 / 3} \simeq 2.4 \times 10^{-4}$ by adopting $\alpha=0.005$ and $h=0.07$ (at the location of the 2:1 MMR with Jupiter). In other words, the planet needs to be no more massive than Saturn to fulfill the above condition. Therefore, the large amplitude one-sided corotation torque condition holds for our considered planet and disk circumstances.

For a general situation, the total torque can be expressed by interpolating the torques in the 
above two regimes

$$
\Gamma=C_{\text {mig }} f \Gamma_{1 \mathrm{~s}}+(1-f) \Gamma_{2 \mathrm{~s}},
$$

where $f=\exp \left[-\left(r_{\mathrm{p}}-r_{\mathrm{in}}\right) / x_{\mathrm{hs}}\right]$ is a smooth fitting function, $r_{\mathrm{p}}$ is the planet disk location, and $f=1(f=0)$ refers to the planet at the disk edge (far away from the disk edge). Although the above equations are derived analytically, we note that the termination of planet migration at the inner disk edge due to one-sided torque induced from a steep density transition is also obtained from hydrodynamic simulations 2526 .

In order to satisfy the planet outward migration at the inner disk edge, the one-sided corotation torque needs to be larger than that the one-sided Lindblad torque. Thus, from Eq. (6) the planet needs to satisfy $q_{\mathrm{p}}<\left(C_{\mathrm{hs}} / C_{\mathrm{L}}\right)^{2} h^{3}$. On the other hand, the above formulas are derived by assuming that the planet torques are in the linear-regime. When the planet is massive enough to progressively clear the local surrounding gas in its horseshoe region, an annular gap can be formed. In this case the disk feedback is non-trivial, and Eqs. (5) and (6) are not applicable anymore. Because the positive corotation torque diminishes due to gap formation, the planet beyond the gap-opening mass fails to undergo outward migration. The gap-opening first requires the planet's Hill sphere $R_{\mathrm{H}} \equiv\left(m_{\mathrm{p}} / 3 M_{\star}\right)^{1 / 3} r$ larger than the disk scale height $H$. Therefore, $q_{\mathrm{p}}<3 h^{3}$ is needed for torques in the linear regime. Dedicated hydrodynamic simulations further indicated that the planet mass needs to fulfill 55

$$
\frac{3}{4} \frac{H}{R_{\mathrm{H}}}+\frac{50}{q_{\mathrm{p}} R_{\mathrm{e}}}>1
$$

where $R_{\mathrm{e}}$ is the Reynolds number $r^{2} \Omega_{\mathrm{K}} / \nu$. We name $q_{\mathrm{p}, \mathrm{c}}$ as the maximum non-gap opening mass ratio obtained from Eq. (8). To summarize, the planet-to-star mass ratio needs to satisfy $q_{\mathrm{p}}<\min \left[\left(C_{\mathrm{hs}} / C_{\mathrm{L}}\right)^{2} h^{3}, 3 h^{3}, q_{\mathrm{p}, \mathrm{c}}\right]$ for planet outward migration at the disk edge.

For our adopted disk model, Saturn, Uranus and Neptune are in the linear type I torque regime. More massive Jupiter is in the type II gap-opening regime, and rebound fails to operate for Jupiter. Since the timescale of type II migration is much longer than that of the type I and our study merely focuses on the time span of rapid disk dispersal, we neglect the migration of Jupiter for simplicity.

Numerical methods. In order to study the evolution of multi-planet systems during gas disk dispersal, we carried out numerical simulations using a modified version of the publicly available $\mathrm{N}$ - 
body code HERMIT4 56 . The code is designed for accurate calculations of both gravitational interactions among multi-bodies and their tidal interactions with the protoplanetary disk gas $24[57$. The gravitational forces among planets are integrated with a time-symmetric scheme and the BurdetHeggie regularization, while the planet-disk interaction is taken into account by implementing the torque recipes mentioned previously. The code enables fast and reliable integration of the orbital evolution of multiple planets associated with close-encounters and collisions for millions of orbits, well suited for this study.

Parameter study. We conduct a high number of numerical simulations to study the evolution of planetary systems during the final gas disk dispersal phase. The initial disk and planet conditions are listed in Table 1. We consider three different planet configurations: all planets in nearly 2:1 resonances, 3:2 resonances and a combination of 2:1 and 3:2 resonances. Different initial numbers of planets have also been explored for $N_{\mathrm{i}}=4,5$ and 6 . In order to study the results in a statistical manner, for each planetary configuration, we have performed $10^{3}$ simulations by Monte Carlo sampling their initial disk conditions $\left(\dot{M}_{\mathrm{pho}}, \tau_{\mathrm{d}}\right.$ and $\left.v_{\mathrm{r}}\right)$ from log-uniform distributions. Besides, we have considered both simulations with and without rebound to evaluate the efficacy of this mechanism. Planets feel the classical type I torques ${ }^{54}$ when rebound is absent, whereas one-sided torque component in Eq. (6) is accounted for when rebound is present. For instance, Figure 2 demonstrates the results of run_B5R/B5N/C5R/C5N in Table 1 .

The initial eccentricities and inclinations of the planets are assumed to follow Rayleigh distributions, where $e_{0}=2 i_{0}=10^{-3}$ is the scale parameter. The other orbital phase angles are randomly distributed between $0^{\circ}$ and $360^{\circ}$. We start the initial planet period ratios $5 \%$ percent higher than the exact resonance values. For the first $0.5 \mathrm{Myr}$, we only turned on the migration of the outer most planet, and remain the cavity and gas surface density unchanged. This ensures the planets get into the desired resonant chains. After that we turn on the mentioned migration prescription and let the disk starts to deplete. Since we only focus on the early instabilities, simulations are run until $t=10$ Myr. It is noteworthy that the assumed values of initial eccentricities barely matter the subsequent evolution since the orbits would quickly get regulated by resonance trapping.

Similar to Figure 2, Figure 4 illustrates the RMC and AMD distributions among surviving planetary systems for initial four-planet systems starting with 3:2 resonances and a chain of 2:1 and $3: 2$ resonances. Figure 5 is for initial four and five planetary systems starting with $2: 1$ resonances, 
Table 1: Initial conditions for a parameter study. The second, third and fourth columns correspond to the initial number, period ratio and the order of the system (from inner to outer), where $\mathrm{J}$, S, U, N are short for the planet with the mass of Jupiter, Saturn, Uranus and Neptune, respectively. The disk parameters $\dot{M}_{\text {pho }}, \tau_{\mathrm{d}}$ and $v_{\mathrm{r}}$ are adopted from log-uniform distributions.

\begin{tabular}{|c|c|c|c|c|c|c|c|}
\hline run ID & $\begin{array}{c}\text { number } \\
N_{\mathrm{i}}\end{array}$ & $\begin{array}{l}\text { period } \\
\text { ratio }\end{array}$ & $\begin{array}{l}\text { mass } \\
\text { order }\end{array}$ & $\begin{array}{l}\text { with } \\
\text { rebound }\end{array}$ & $\begin{array}{c}\dot{M}_{\mathrm{pho}} \\
{\left[\mathrm{M}_{\odot} \mathrm{yr}^{-1}\right]}\end{array}$ & $\begin{array}{l}\tau_{\mathrm{d}} \\
{[\mathrm{yr}]}\end{array}$ & $\begin{array}{c}v_{\mathrm{r}} \\
{\left[\mathrm{AU} \mathrm{Myr}^{-1}\right]}\end{array}$ \\
\hline run $A 4 R$ & 4 & all $2: 1$ & $\mathrm{~J}, \mathrm{~S}, \mathrm{U}, \mathrm{N}$ & Yes & {$\left[10^{-9.5}, 10^{-8.5}\right]$} & {$\left[10^{5}, 10^{6}\right]$} & {$[20,200]$} \\
\hline $\operatorname{run} A 4 N$ & 4 & all $2: 1$ & $\mathrm{~J}, \mathrm{~S}, \mathrm{U}, \mathrm{N}$ & No & {$\left[10^{-9.5}, 10^{-8.5}\right]$} & {$\left[10^{5}, 10^{6}\right]$} & {$[20,200]$} \\
\hline run $B 4 R$ & 4 & all $3: 2$ & $\mathrm{~J}, \mathrm{~S}, \mathrm{U}, \mathrm{N}$ & Yes & {$\left[10^{-9.5}, 10^{-8.5}\right]$} & {$\left[10^{5}, 10^{6}\right]$} & {$[20,200]$} \\
\hline run $B 4 N$ & 4 & all $3: 2$ & $\mathrm{~J}, \mathrm{~S}, \mathrm{U}, \mathrm{N}$ & No & {$\left[10^{-9.5}, 10^{-8.5}\right]$} & {$\left[10^{5}, 10^{6}\right]$} & {$[20,200]$} \\
\hline run_C4R & 4 & $2: 1,3: 2,3: 2,3: 2$ & $\mathrm{~J}, \mathrm{~S}, \mathrm{U}, \mathrm{N}$ & Yes & {$\left[10^{-9.5}, 10^{-8.5}\right]$} & {$\left[10^{5}, 10^{6}\right]$} & {$[20,200]$} \\
\hline run_C4N & 4 & $2: 1,3: 2,3: 2,3: 2$ & $\mathrm{~J}, \mathrm{~S}, \mathrm{U}, \mathrm{N}$ & No & {$\left[10^{-9.5}, 10^{-8.5}\right]$} & {$\left[10^{5}, 10^{6}\right]$} & {$[20,200]$} \\
\hline run $A 5 R$ & 5 & all $2: 1$ & $\mathrm{~J}, \mathrm{~S}, \mathrm{U}, \mathrm{U}, \mathrm{N}$ & Yes & {$\left[10^{-9.5}, 10^{-8.5}\right]$} & {$\left[10^{5}, 10^{6}\right]$} & {$[20,200]$} \\
\hline $\operatorname{run} A 5 N$ & 5 & all $2: 1$ & $\mathrm{~J}, \mathrm{~S}, \mathrm{U}, \mathrm{U}, \mathrm{N}$ & No & {$\left[10^{-9.5}, 10^{-8.5}\right]$} & {$\left[10^{5}, 10^{6}\right]$} & {$[20,200]$} \\
\hline run_B5R & 5 & all $3: 2$ & $\mathrm{~J}, \mathrm{~S}, \mathrm{U}, \mathrm{U}, \mathrm{N}$ & Yes & {$\left[10^{-9.5}, 10^{-8.5}\right]$} & {$\left[10^{5}, 10^{6}\right]$} & {$[20,200]$} \\
\hline run_B5N & 5 & all $3: 2$ & $\mathrm{~J}, \mathrm{~S}, \mathrm{U}, \mathrm{U}, \mathrm{N}$ & No & {$\left[10^{-9.5}, 10^{-8.5}\right]$} & {$\left[10^{5}, 10^{6}\right]$} & {$[20,200]$} \\
\hline run_C5R & 5 & $2: 1,3: 2,3: 2,3: 2$ & $\mathrm{~J}, \mathrm{~S}, \mathrm{U}, \mathrm{U}, \mathrm{N}$ & Yes & {$\left[10^{-9.5}, 10^{-8.5}\right]$} & {$\left[10^{5}, 10^{6}\right]$} & {$[20,200]$} \\
\hline run_C5N & 5 & $2: 1,3: 2,3: 2,3: 2$ & $\mathrm{~J}, \mathrm{~S}, \mathrm{U}, \mathrm{U}, \mathrm{N}$ & No & {$\left[10^{-9.5}, 10^{-8.5}\right]$} & {$\left[10^{5}, 10^{6}\right]$} & {$[20,200]$} \\
\hline run $B 6 R$ & 6 & all $3: 2$ & $\mathrm{~J}, \mathrm{~S}, \mathrm{U}, \mathrm{U}, \mathrm{N}, \mathrm{N}$ & Yes & {$\left[10^{-9.5}, 10^{-8.5}\right]$} & {$\left[10^{5}, 10^{6}\right]$} & {$[20,200]$} \\
\hline run_C6R & 6 & $2: 1,3: 2,3: 2,3: 2$ & $\mathrm{~J}, \mathrm{~S}, \mathrm{U}, \mathrm{U}, \mathrm{N}, \mathrm{N}$ & Yes & {$\left[10^{-9.5}, 10^{-8.5}\right]$} & {$\left[10^{5}, 10^{6}\right]$} & {$[20,200]$} \\
\hline
\end{tabular}

while Figure 6 is for initial six-planet systems. The crucial influence of rebound during disk dispersal on final architecture of the systems is clear present in these figures as well. In addition, the AMDs of systems with initial four, five and six planets as a function of disk parameters are presented in Figures 7, 8 and 9, respectively. The model parameter setups and numerical outcomes can be found in Table 1 and Table 2 .

\section{Data availability}

The data that support the plots within this paper and other findings of this study are available from the corresponding author upon reasonable request. 
Table 2: Simulation results for a parameter study. The second, third, fourth and fifth columns show the probability of systems with final number of planets $N_{\mathrm{f}}=6,5,4$ and $\leqslant 3$, respectively. The sixth column represents the probability of forming Solar System analogs, defined as systems with four surviving planets with right orders and their AMDs and RMCs within a factor of three compared to the Solar System values.

\begin{tabular}{lclclc}
\hline \hline run ID & $p_{N_{\mathrm{f}}=6}$ & $p_{N_{\mathrm{f}}=5}$ & $p_{N_{\mathrm{f}}=4}$ & $p_{N_{\mathrm{f}}<=3}$ & $p_{\mathrm{SSA}}$ \\
\hline run_A4R & $0 \%$ & $0 \%$ & $73.6 \%$ & $26.4 \%$ & $7.7 \%$ \\
run_A4N & $0 \%$ & $0 \%$ & $100.0 \%$ & $0.0 \%$ & $89.7 \%$ \\
run_B4R & $0 \%$ & $0 \%$ & $23.1 \%$ & $76.9 \%$ & $0.9 \%$ \\
run_B4N & $0 \%$ & $0 \%$ & $84.5 \%$ & $15.5 \%$ & $0.0 \%$ \\
run_C4R & $0 \%$ & $0 \%$ & $45.1 \%$ & $54.9 \%$ & $6.4 \%$ \\
run_C4N & $0 \%$ & $0 \%$ & $77.9 \%$ & $22.1 \%$ & $58.8 \%$ \\
run_A5R & $0 \%$ & $69.3 \%$ & $26.9 \%$ & $3.8 \%$ & $1.7 \%$ \\
run_A5N & $0 \%$ & $100.0 \%$ & $0.0 \%$ & $0.0 \%$ & $0.0 \%$ \\
run_B5R & $0 \%$ & $7.1 \%$ & $29.4 \%$ & $63.5 \%$ & $1.9 \%$ \\
run_B5N & $0 \%$ & $61.2 \%$ & $7.3 \%$ & $31.5 \%$ & $2.4 \%$ \\
run_C5R & $0 \%$ & $22.3 \%$ & $40.6 \%$ & $36.1 \%$ & $2.5 \%$ \\
run_C5N & $0 \%$ & $69.1 \%$ & $10.7 \%$ & $20.2 \%$ & $3.3 \%$ \\
run_B6R & $3.1 \%$ & $19.1 \%$ & $39.7 \%$ & $38.1 \%$ & $1.4 \%$ \\
run_C6R & $15.4 \%$ & $30.1 \%$ & $37.9 \%$ & $16.6 \%$ & $0.3 \%$ \\
\hline \hline
\end{tabular}




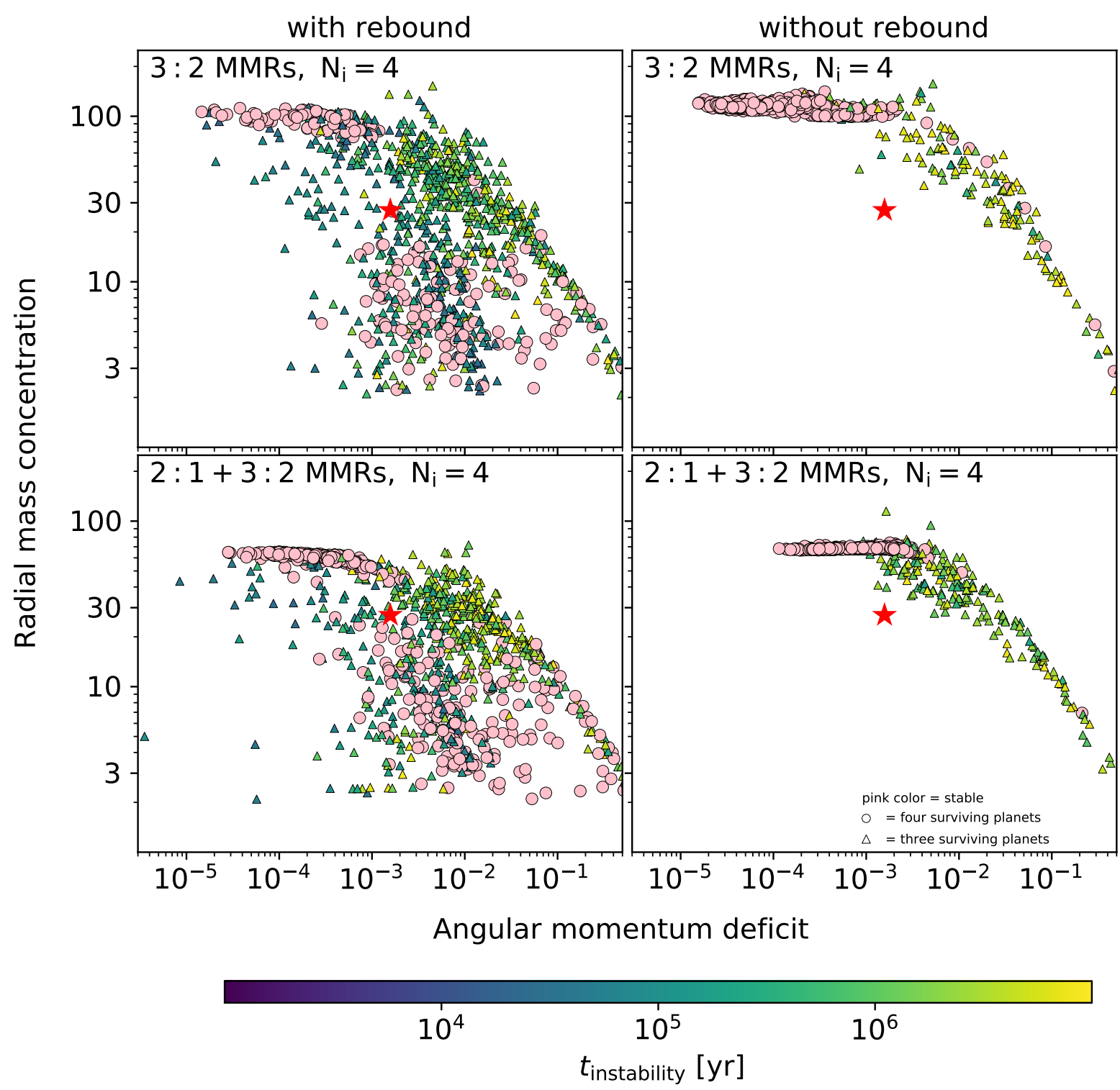

Figure 4: The radial mass concentration and normalized angular momentum deficit distributions among surviving planetary systems in our simulations. The simulations on the left included the rebound effect and those on the right did not. For this subset of our simulations, the four giant planets were included, initially in a chain of 3:2 (top) or a combination of 2:1 and 3:2 (bottom) orbital resonances. Circles and triangles correspond to systems with four and three or fewer surviving planets, respectively. The pink refers to stable systems while the color bar indicates the time when the instability occurs after the start of gas disk dispersal. The Solar System is marked as a red star for comparison. 

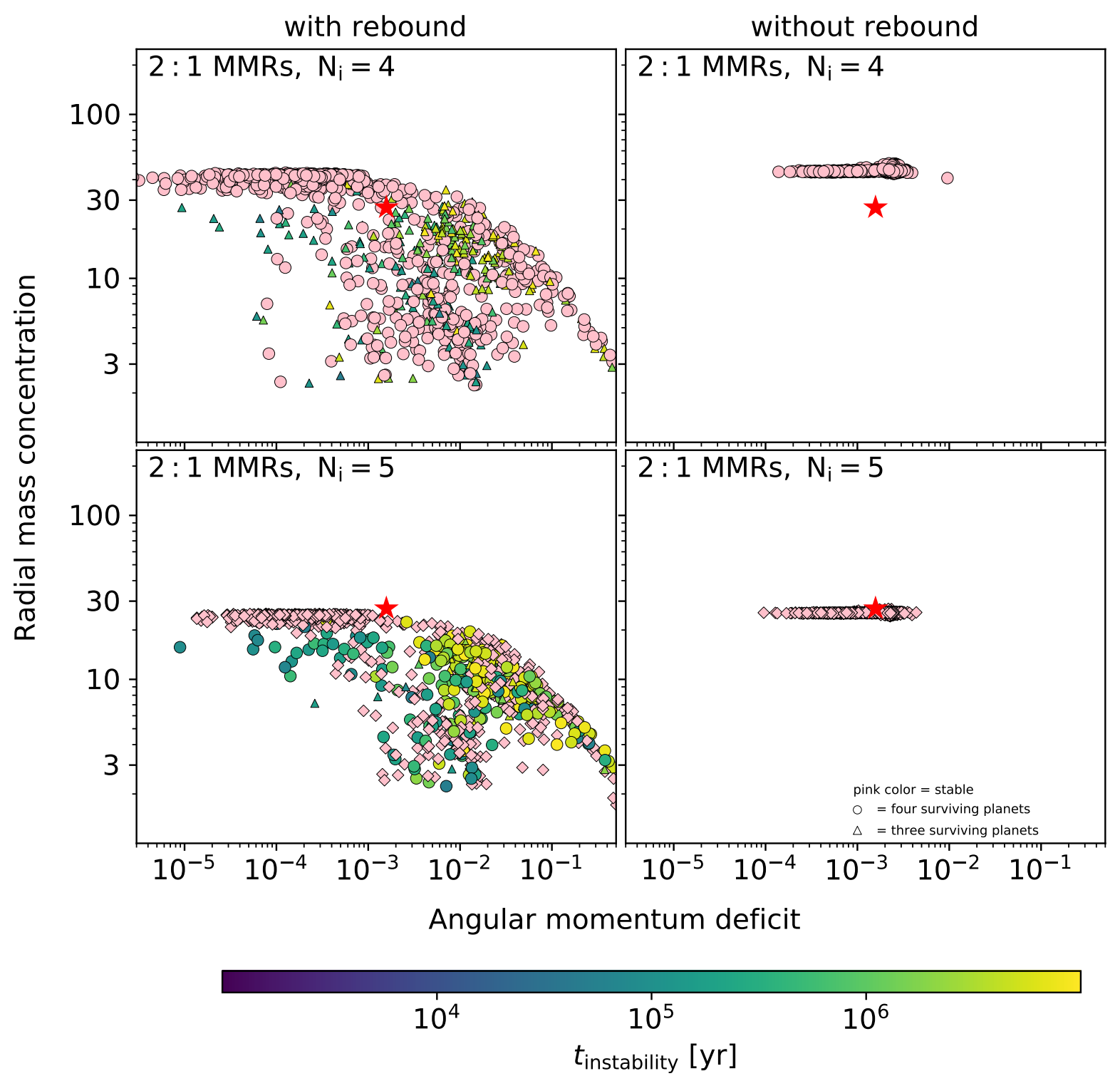

Figure 5: The radial mass concentration and normalized angular momentum deficit distributions among surviving planetary systems in our simulations. The simulations on the left included the rebound effect and those on the right did not. Systems are started with initial four (top) and five giant planets (bottom) in a chain of 2:1 orbital resonances. Squares, circles and triangles correspond to systems with five, four and three or fewer surviving planets, respectively. The pink refers to the system without any dynamical instability (no collision/ejection), while the color bar indicates the time when the instability occurs after the start of gas disk dispersal. The Solar System is marked as a red star for comparison. 


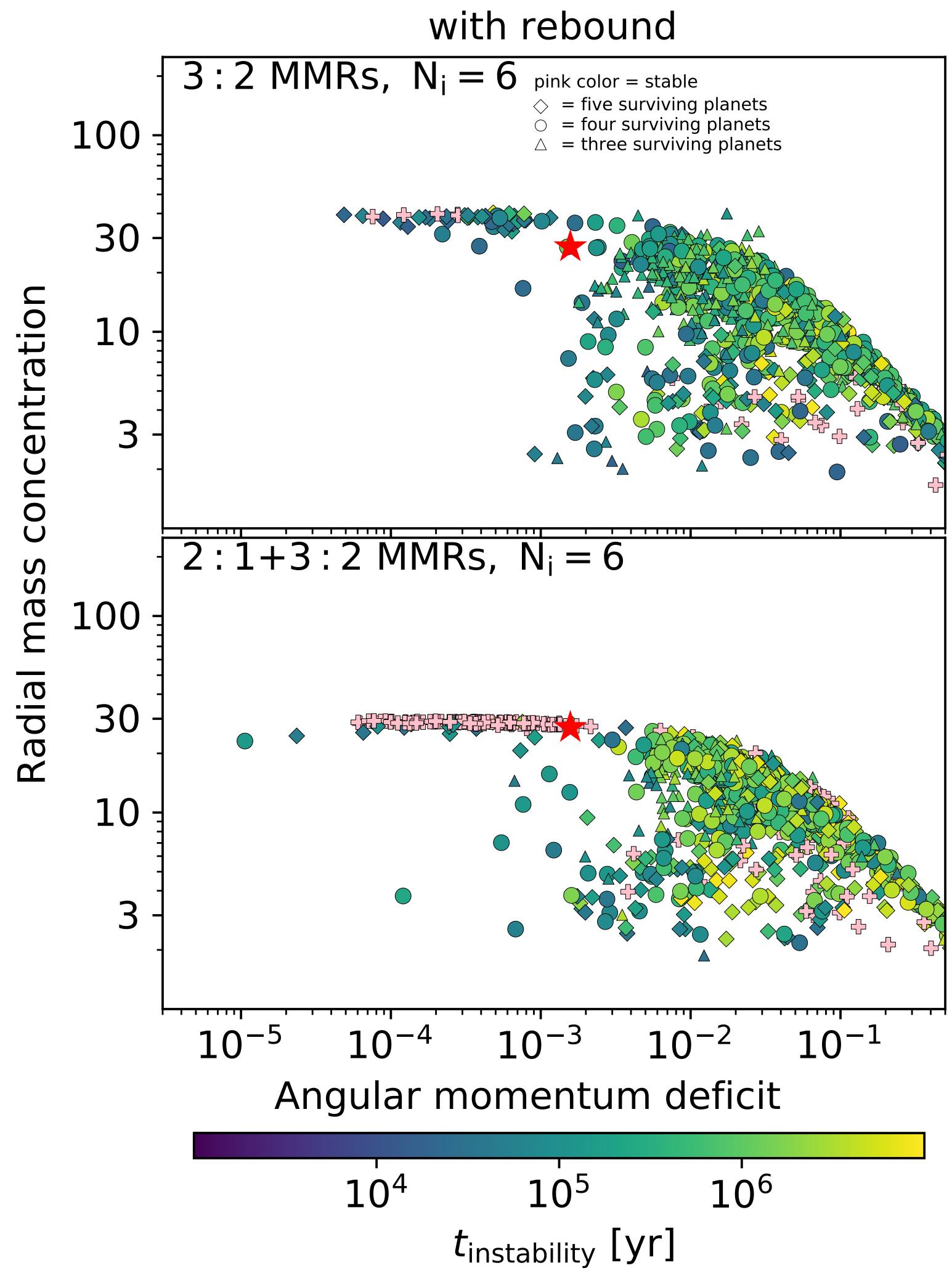

Figure 6: The radial mass concentration and normalized angular momentum deficit distributions among surviving planetary systems in our simulations. For this subset of our simulations, the six giant planets were included, initially in a chain of 2:1 (top) or a combination of $2: 1$ and 3:2 (bottom) orbital resonances. Plus, squares, circles and triangles correspond to systems with six, five, four and three or fewer surviving planets, respectively.The pink refers to the system without 

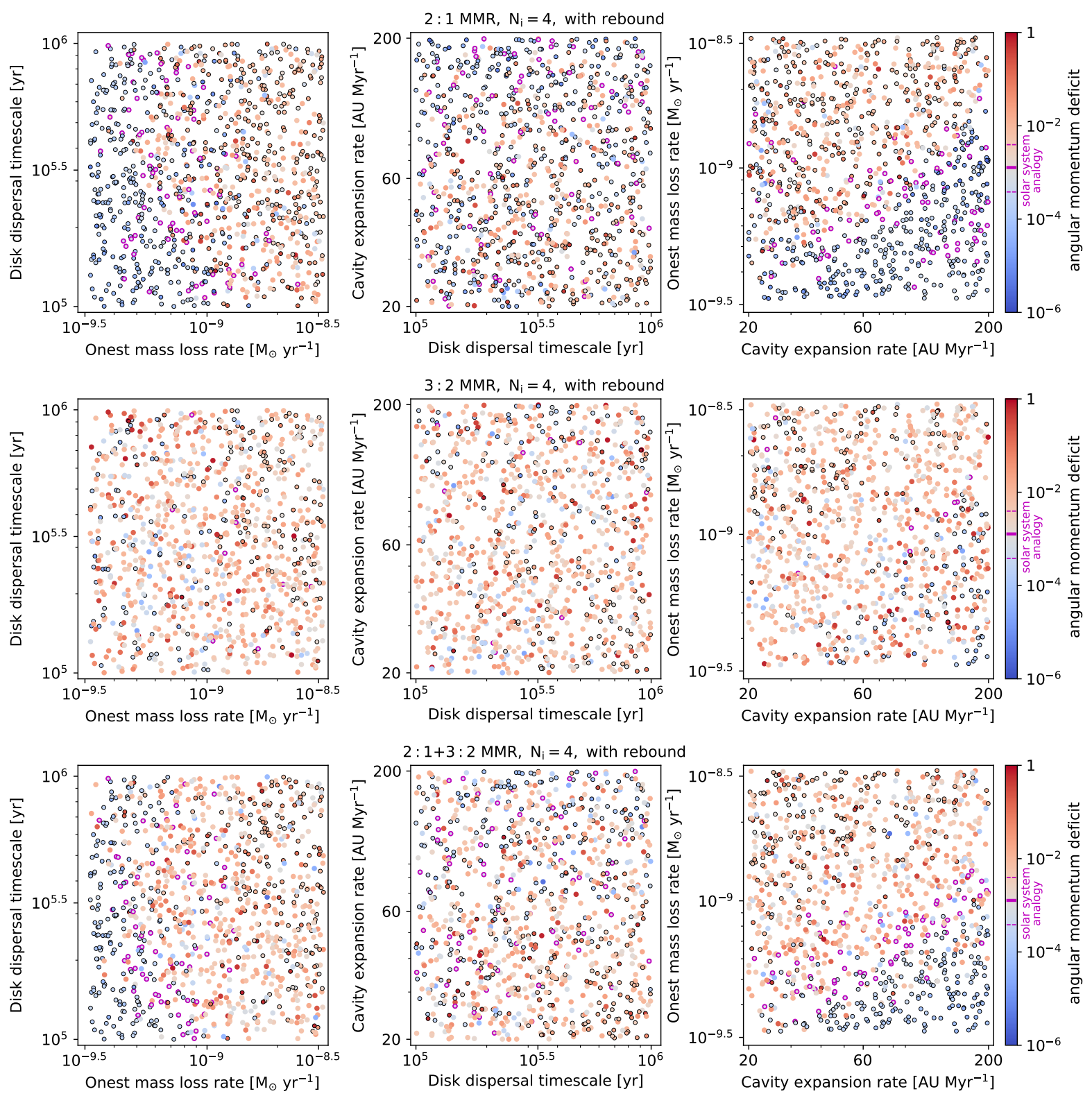

Figure 7: Simulation outcomes of systems with initial four planets as a function of disk parameters. Each simulation started with our four present-day giant planets in a series of 2:1 (top) and 3:2 (middle) and a combination of 2:1 and 3:2 (bottom) orbital resonances. The color bar corresponds to the system's angular momentum deficit AMD. The circles with black edgecolor refer to the systems whose planets all survive in the end, while the circles with magenta edgecolor represent the Solar System analogs, defined as systems with final four surviving planets with right orders and their AMDs and RMCs are within a factor of three compared to those of our Solar System. 

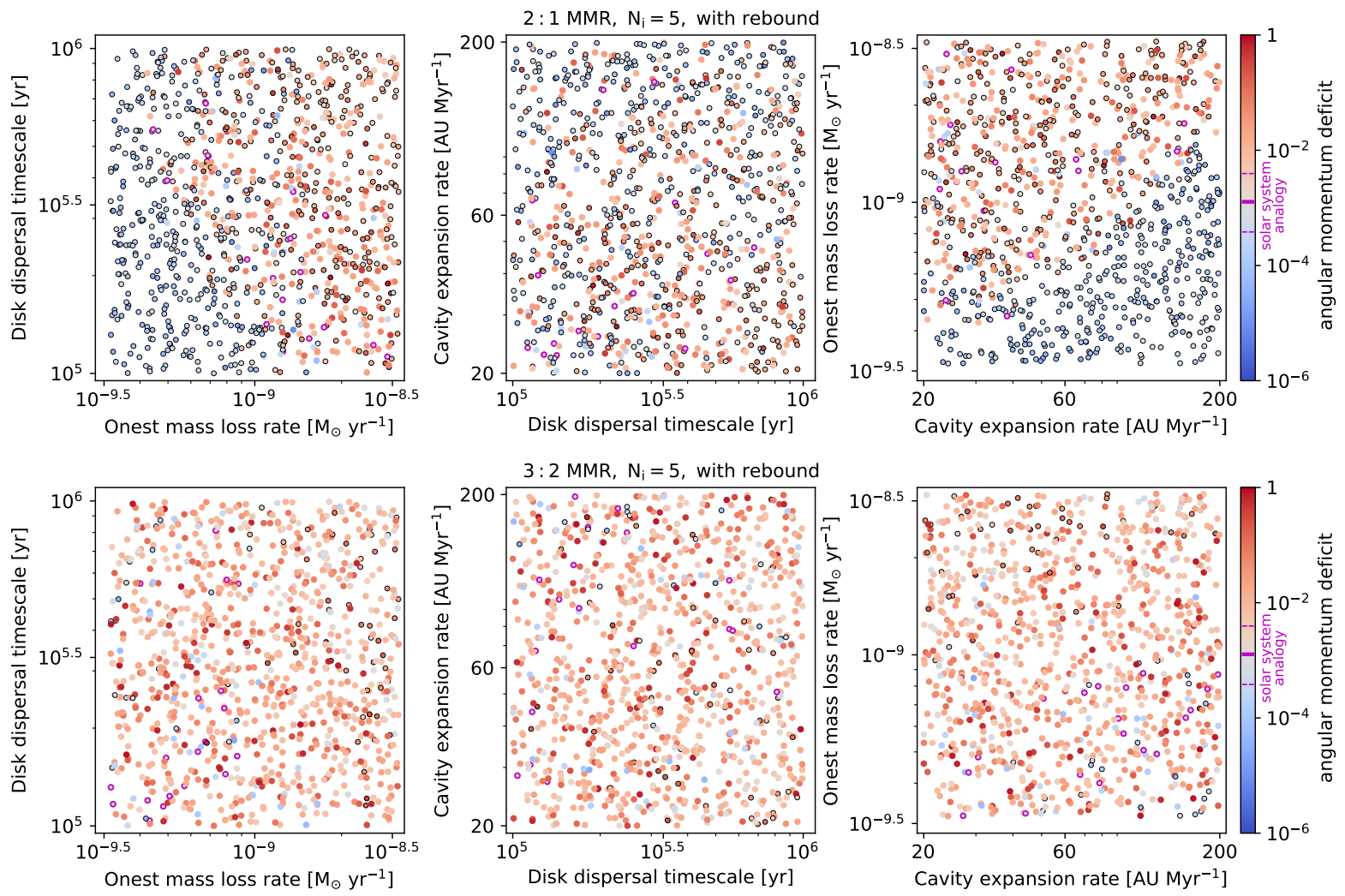

Figure 8: Simulation outcomes of systems with initial five planets as a function of disk parameters. Each simulation started with our four present-day giant planets plus one additional ice giant planet in a series of 2:1 (top), 3:2 (bottom) orbital resonances. The color bar corresponds to the system's angular momentum deficit AMD. The circles with black edgecolor refer to the systems whose planets all survive in the end, while the circles with magenta edgecolor represent the Solar System analogs, defined as systems with four surviving planets with right orders and their AMDs and RMCs are within a factor of three compared to those of our Solar System. 

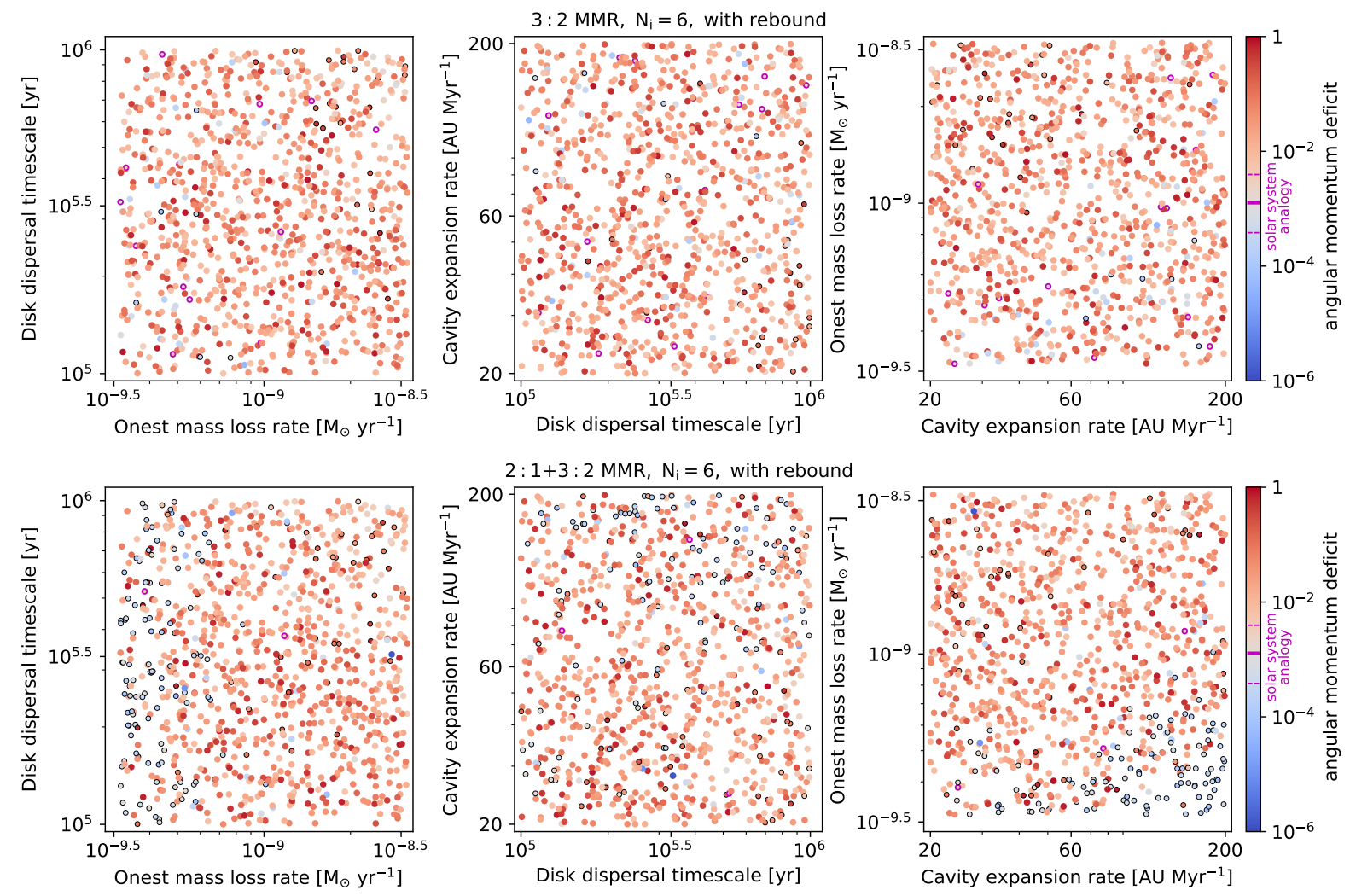

Figure 9: Simulation outcomes of systems with initial six planets as a function of disk parameters. Each simulation started with our four present-day giant planets plus two additional ice giant planet in a series of 2:1 (top) and a combination of 2:1 and 3:2 (bottom) orbital resonances. The color bar corresponds to the system's angular momentum deficit AMD. The circles with black edgecolor refer to the systems whose planets all survive in the end, while the circles with magenta edgecolor represent the Solar System analogs, defined as systems with four surviving planets with right orders and their AMDs and RMCs are within a factor of three compared to those of our Solar System. 


\section{Code availability}

The source code and simulation output for the model used in this study are available on request from the corresponding authors. The original version of the HERMIT4 N-body code is publicly available in Sverre Aarseth's homepage https://people.ast.cam.ac.uk/ sverre/ web/pages/nbody . htm. 
1. Tsiganis, K., Gomes, R., Morbidelli, A. \& Levison, H. F. Origin of the orbital architecture of the giant planets of the Solar System. Nature 435, 459-461 (2005).

2. Morbidelli, A., Tsiganis, K., Crida, A., Levison, H. F. \& Gomes, R. Dynamics of the Giant Planets of the Solar System in the Gaseous Protoplanetary Disk and Their Relationship to the Current Orbital Architecture. Astron. J. 134, 1790-1798 (2007).

3. Batygin, K., Brown, M. E. \& Betts, H. Instability-driven Dynamical Evolution Model of a Primordially Five-planet Outer Solar System. Astrophys. J. Lett. 744, L3 (2012).

4. Nesvorný, D. Dynamical Evolution of the Early Solar System. Ann. Rev. Astron. Astrophys. 56, 137-174 (2018).

5. Levison, H. F., Morbidelli, A., Tsiganis, K., Nesvorný, D. \& Gomes, R. Late Orbital Instabilities in the Outer Planets Induced by Interaction with a Self-gravitating Planetesimal Disk. Astron. J. 142, 152 (2011).

6. Nesvorný, D., Vokrouhlický, D., Bottke, W. F. \& Levison, H. F. Evidence for very early migration of the Solar System planets from the Patroclus-Menoetius binary Jupiter Trojan. Nat. Astron. 2, 878-882 (2018).

7. Quarles, B. \& Kaib, N. Instabilities in the Early Solar System Due to a Self-gravitating Disk. Astron. J. 157, 67 (2019).

8. Mojzsis, S. J., Brasser, R., Kelly, N. M., Abramov, O. \& Werner, S. C. Onset of Giant Planet Migration before 4480 Million Years Ago. Astrophys. J. 881, 44 (2019).

9. de Sousa, R. R. et al. Dynamical evidence for an early giant planet instability. Icarus 339, 113605 (2020).

10. Rivera, E. J. et al. The Lick-Carnegie Exoplanet Survey: a Uranus-Mass Fourth Planet for GJ 876 in an Extrasolar Laplace Configuration. Astrophys. J. 719, 890-899 (2010).

11. Pierens, A., Raymond, S. N., Nesvorny, D. \& Morbidelli, A. Outward Migration of Jupiter and Saturn in 3:2 or 2:1 Resonance in Radiative Disks: Implications for the Grand Tack and Nice models. Astrophys. J. Lett. 795, L11 (2014). 
12. Williams, J. P. \& Cieza, L. A. Protoplanetary Disks and Their Evolution. Ann. Rev. Astron. Astrophys. 49, 67-117 (2011).

13. Touboul, M., Kleine, T., Bourdon, B., Palme, H. \& Wieler, R. Late formation and prolonged differentiation of the Moon inferred from W isotopes in lunar metals. Nature 450, 1206-1209 (2007).

14. Jacobson, S. A. et al. Highly siderophile elements in Earth's mantle as a clock for the Moonforming impact. Nature 508, 84-87 (2014).

15. Kaib, N. A. \& Chambers, J. E. The fragility of the terrestrial planets during a giant-planet instability. Mon. Not. R. Astron. Soc. 455, 3561-3569 (2016).

16. Clement, M. S., Kaib, N. A., Raymond, S. N. \& Walsh, K. J. Mars' growth stunted by an early giant planet instability. Icarus 311, 340-356 (2018).

17. Armitage, P. J. Dynamics of Protoplanetary Disks. Ann. Rev. Astron. Astrophys. 49, 195-236 (2011).

18. Alexander, R., Pascucci, I., Andrews, S., Armitage, P. \& Cieza, L. The Dispersal of Protoplanetary Disks. In Beuther, H., Klessen, R. S., Dullemond, C. P. \& Henning, T. (eds.) Protostars and Planets VI, 475 (2014).

19. Alexander, R. D., Clarke, C. J. \& Pringle, J. E. Photoevaporation of protoplanetary discs - I. Hydrodynamic models. Mon. Not. R. Astron. Soc. 369, 216-228 (2006).

20. Espaillat, C. et al. An Observational Perspective of Transitional Disks. In Beuther, H., Klessen, R. S., Dullemond, C. P. \& Henning, T. (eds.) Protostars and Planets VI, 497 (2014).

21. Adams, F. C., Hollenbach, D., Laughlin, G. \& Gorti, U. Photoevaporation of Circumstellar Disks Due to External Far-Ultraviolet Radiation in Stellar Aggregates. Astrophys. J. 611, 360-379 (2004).

22. Goldreich, P. \& Tremaine, S. Disk-satellite interactions. Astrophys. J. 241, 425-441 (1980).

23. Cresswell, P. \& Nelson, R. P. Three-dimensional simulations of multiple protoplanets embedded in a protostellar disc. Astron. Astrophys. 482, 677-690 (2008). 
24. Liu, B., Ormel, C. W. \& Lin, D. N. C. Dynamical rearrangement of super-Earths during disk dispersal. I. Outline of the magnetospheric rebound model. Astron. Astrophys. 601, A15 (2017).

25. Masset, F. S., Morbidelli, A., Crida, A. \& Ferreira, J. Disk Surface Density Transitions as Protoplanet Traps. Astrophys. J. 642, 478-487 (2006).

26. Romanova, M. M. et al. 3D simulations of planet trapping at disc-cavity boundaries. Mon. Not. R. Astron. Soc. 485, 2666-2680 (2019).

27. Liu, B. \& Ormel, C. W. Dynamical rearrangement of super-Earths during disk dispersal. II. Assessment of the magnetospheric rebound model for planet formation scenarios. Astron. Astrophys. 606, A66 (2017).

28. Levison, H. F., Morbidelli, A., Van Laerhoven, C., Gomes, R. \& Tsiganis, K. Origin of the structure of the Kuiper belt during a dynamical instability in the orbits of Uranus and Neptune. Icarus 196, 258-273 (2008).

29. Morbidelli, A., Levison, H. F., Tsiganis, K. \& Gomes, R. Chaotic capture of Jupiter's Trojan asteroids in the early Solar System. Nature 435, 462-465 (2005).

30. Nesvorný, D., Vokrouhlický, D. \& Morbidelli, A. Capture of Irregular Satellites during Planetary Encounters. Astron. J. 133, 1962-1976 (2007).

31. Gomes, R., Levison, H. F., Tsiganis, K. \& Morbidelli, A. Origin of the cataclysmic Late Heavy Bombardment period of the terrestrial planets. Nature 435, 466-469 (2005).

32. Morbidelli, A. \& Nesvorný, D. Kuiper belt: formation and evolution. (Elsevier, 2020).

33. Nesvorný, D. \& Morbidelli, A. Statistical Study of the Early Solar System's Instability with Four, Five, and Six Giant Planets. Astron. J. 144, 117 (2012).

34. Clement, M. S. et al. Born eccentric: Constraints on Jupiter and Saturn's pre-instability orbits. Icarus 355, 114122 (2021).

35. Laskar, J. Large scale chaos and the spacing of the inner planets. Astron. Astrophys. 317, L75-L78 (1997). 
36. Chambers, J. E. Making More Terrestrial Planets. Icarus 152, 205-224 (2001).

37. Krot, A. N., Amelin, Y., Cassen, P. \& Meibom, A. Young chondrules in CB chondrites from a giant impact in the early Solar System. Nature 436, 989-992 (2005).

38. Zellner, N. E. B. Cataclysm No More: New Views on the Timing and Delivery of Lunar Impactors. Origins of Life and Evolution of the Biosphere 47, 261-280 (2017).

39. Morbidelli, A. et al. The timeline of the lunar bombardment: Revisited. Icarus 305, 262-276 (2018).

40. Zahnle, K., Schenk, P., Levison, H. \& Dones, L. Cratering rates in the outer Solar System. Icarus 163, 263-289 (2003).

41. Singer, K. N. et al. Impact craters on Pluto and Charon indicate a deficit of small Kuiper belt objects. Science 363, 955-959 (2019).

42. Raymond, S. N., Izidoro, A. \& Morbidelli, A. Solar System Formation in the Context of Extra-Solar Planets. arXiv e-prints (2018).

43. Raymond, S. N., Armitage, P. J. \& Gorelick, N. Planet-Planet Scattering in Planetesimal Disks. II. Predictions for Outer Extrasolar Planetary Systems. Astrophys. J. 711, 772-795 (2010).

44. Izidoro, A. et al. Breaking the chains: hot super-Earth systems from migration and disruption of compact resonant chains. Mon. Not. R. Astron. Soc. 470, 1750-1770 (2017).

45. Ercolano, B. \& Pascucci, I. The dispersal of planet-forming discs: theory confronts observations. Royal Society Open Science 4, 170114 (2017).

46. Shakura, N. I. \& Sunyaev, R. A. Black holes in binary systems. Observational appearance. Astron. Astrophys. 500, 33-51 (1973).

47. Owen, J. E., Ercolano, B. \& Clarke, C. J. Protoplanetary disc evolution and dispersal: the implications of X-ray photoevaporation. Mon. Not. R. Astron. Soc. 412, 13-25 (2011).

48. Haisch, J., Karl E., Lada, E. A. \& Lada, C. J. Disk Frequencies and Lifetimes in Young Clusters. Astrophys. J. Lett. 553, L153-L156 (2001). 
49. Mamajek, E. E. Initial Conditions of Planet Formation: Lifetimes of Primordial Disks. In Usuda, T., Tamura, M. \& Ishii, M. (eds.) American Institute of Physics Conference Series, vol. 1158 of American Institute of Physics Conference Series, 3-10 (2009).

50. Wolk, S. J. \& Walter, F. M. A Search for Protoplanetary Disks Around Naked T Tauri Stars. Astron. J. 111, 2066 (1996).

51. Luhman, K. L., Allen, P. R., Espaillat, C., Hartmann, L. \& Calvet, N. The Disk Population of the Taurus Star-Forming Region. Astrophys. J. Suppl. 186, 111-174 (2010).

52. Koepferl, C. M. et al. Disc clearing of young stellar objects: evidence for fast inside-out dispersal. Mon. Not. R. Astron. Soc. 428, 3327-3354 (2013).

53. Hayashi, C. Structure of the Solar Nebula, Growth and Decay of Magnetic Fields and Effects of Magnetic and Turbulent Viscosities on the Nebula. Progress of Theoretical Physics Supplement 70, 35-53 (1981).

54. Paardekooper, S. J., Baruteau, C., Crida, A. \& Kley, W. A torque formula for non-isothermal type I planetary migration - I. Unsaturated horseshoe drag. Mon. Not. R. Astron. Soc. 401, 1950-1964 (2010).

55. Crida, A., Morbidelli, A. \& Masset, F. On the width and shape of gaps in protoplanetary disks. Icarus 181, 587-604 (2006).

56. Aarseth, S. J. Gravitational N-Body Simulations. (Cambridge University Press, 2003).

57. Liu, B., Zhang, X., Lin, D. N. C. \& Aarseth, S. J. Migration and Growth of Protoplanetary Embryos. II. Emergence of Proto-Gas-Giant Cores versus Super Earth Progenitors. Astrophys. J. 798, 62 (2015). 
Figures

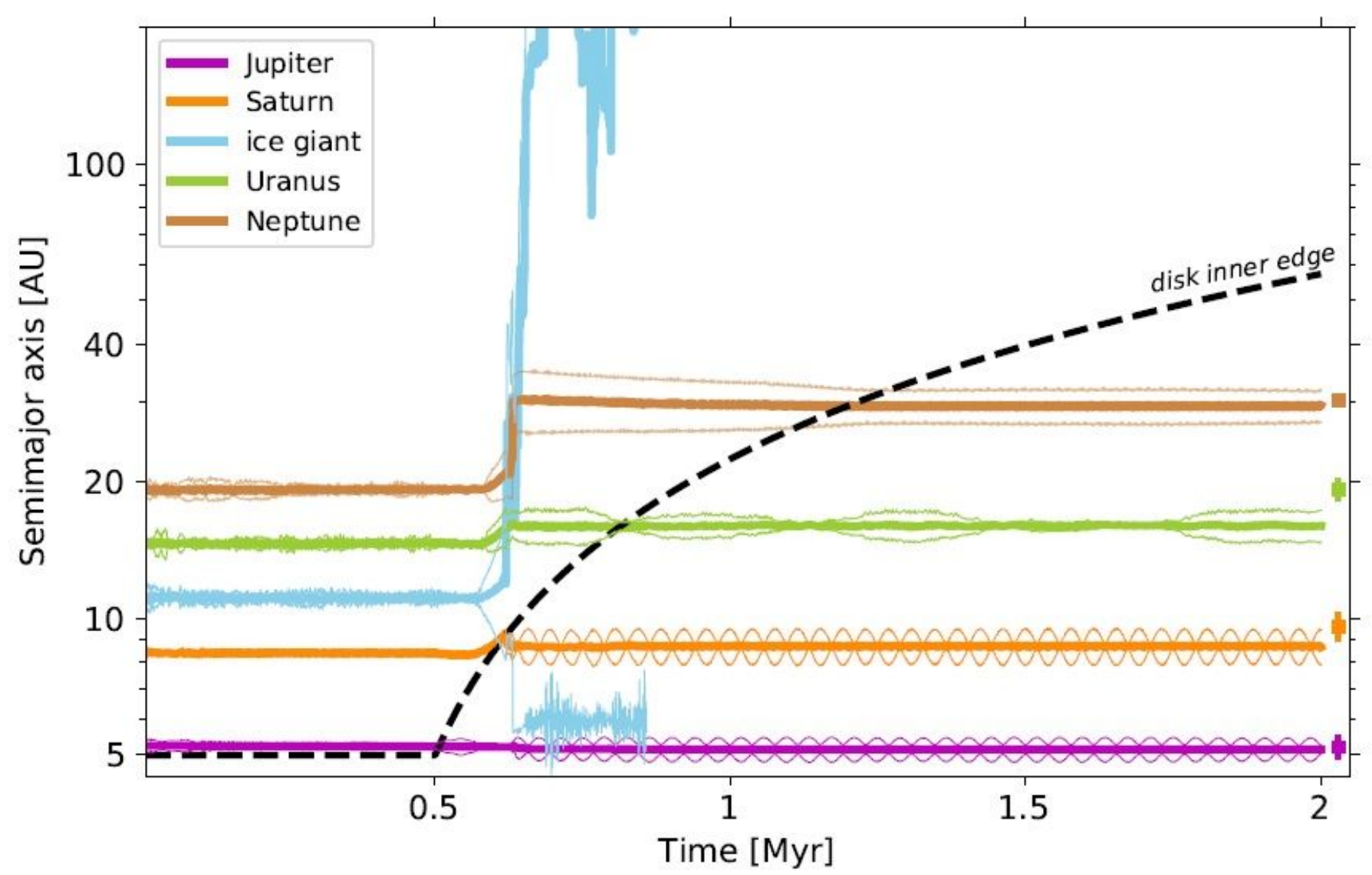

Figure 1

An early dynamical instability triggered by the dispersal of the Sun's protoplanetary disk. (see Manuscript file for full caption) 


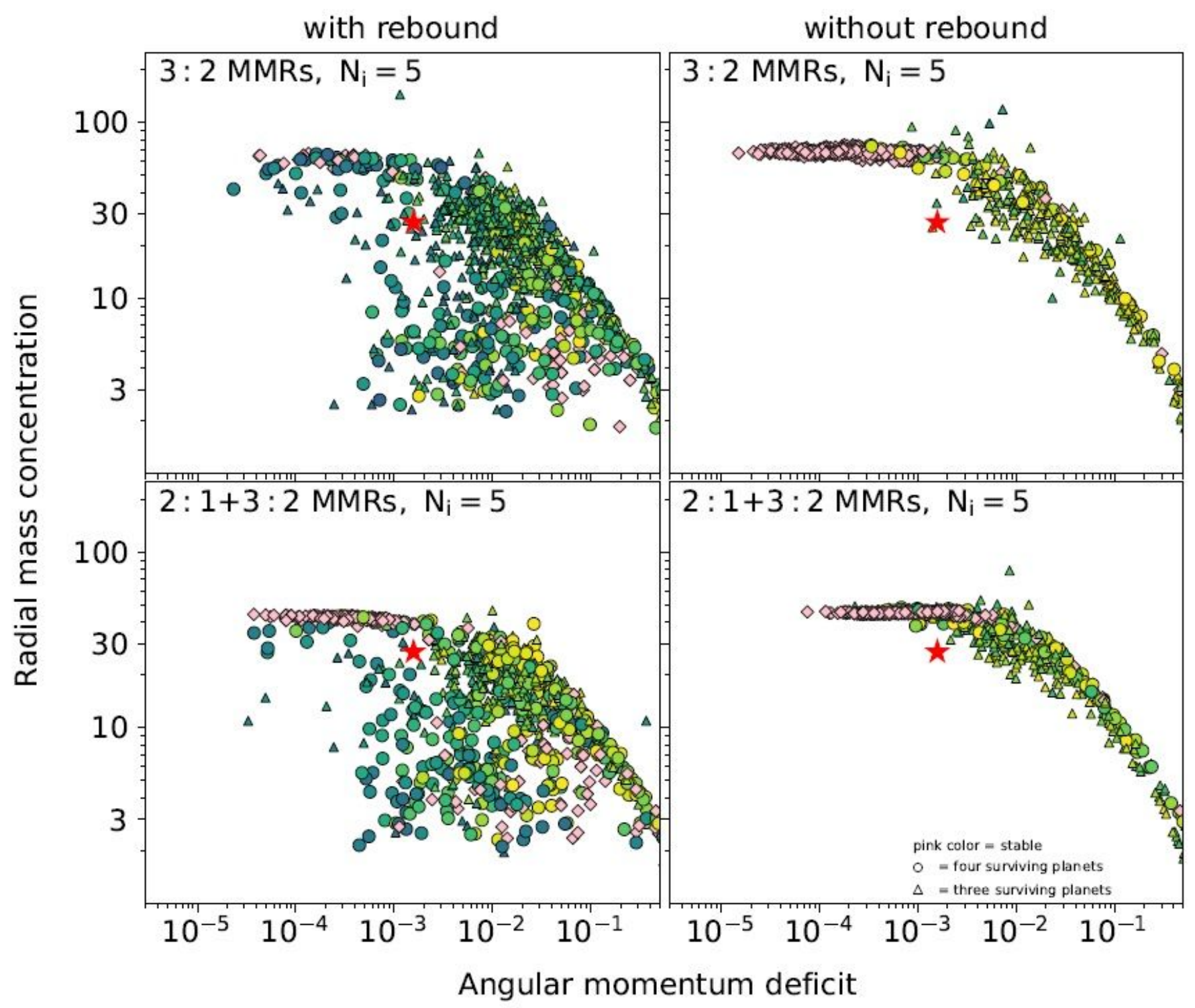

Figure 2

The radial mass concentration (RMC) and normalized angular momentum deficit (AMD) distributions among surviving planetary systems in a subsample of our simulations. (see Manuscript file for full caption) 

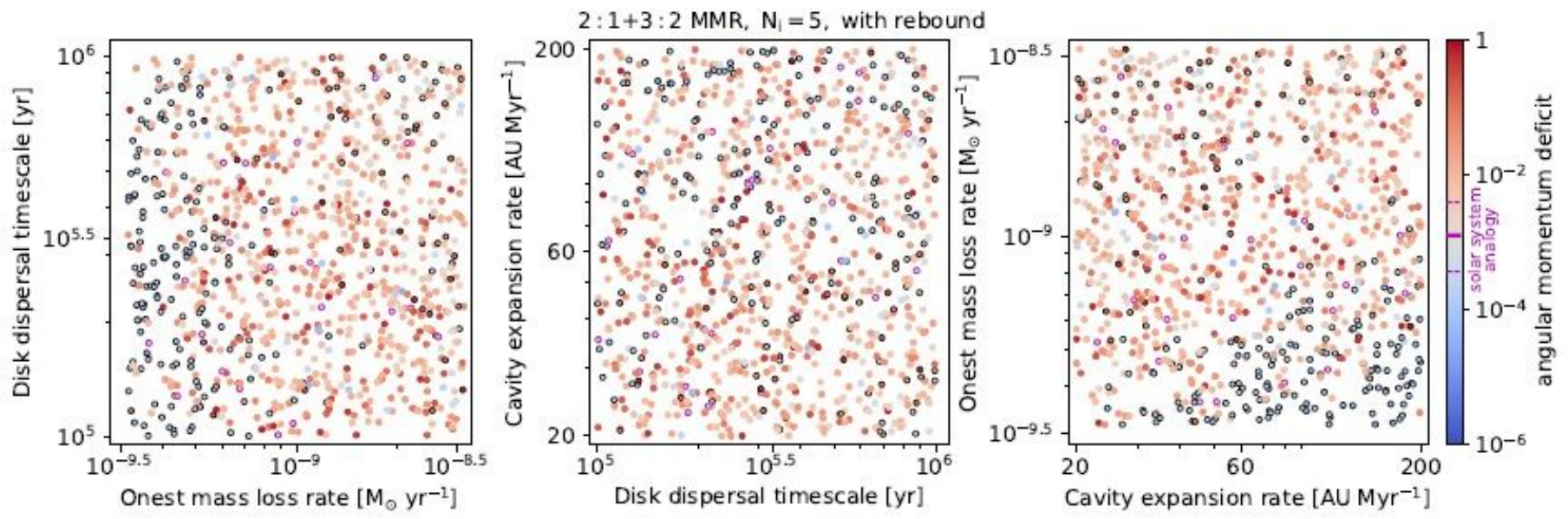

Figure 3

Simulation outcomes as a function of disk parameters. (see Manuscript file for full caption) 


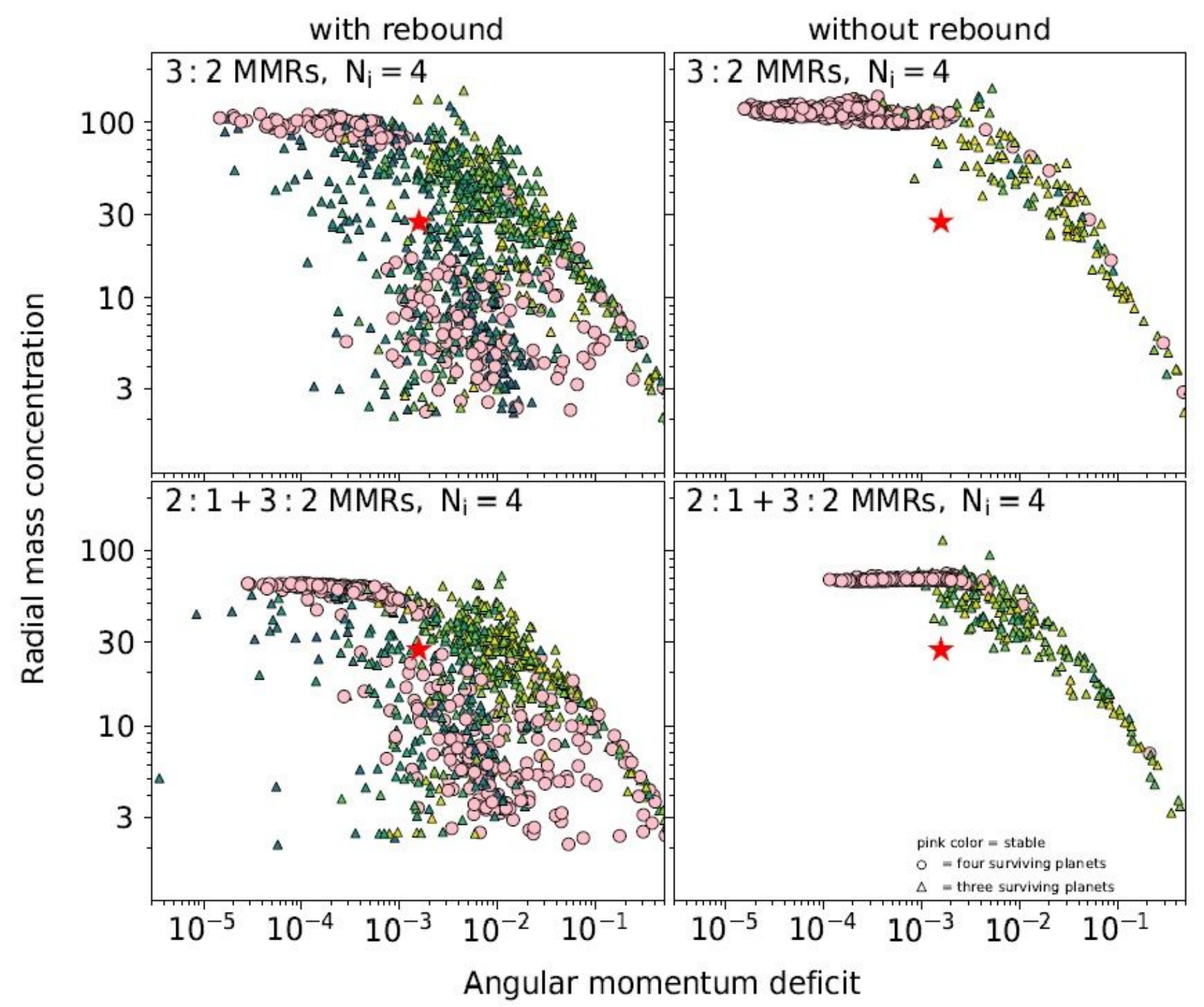

Figure 4

(see Manuscript file for full caption) 

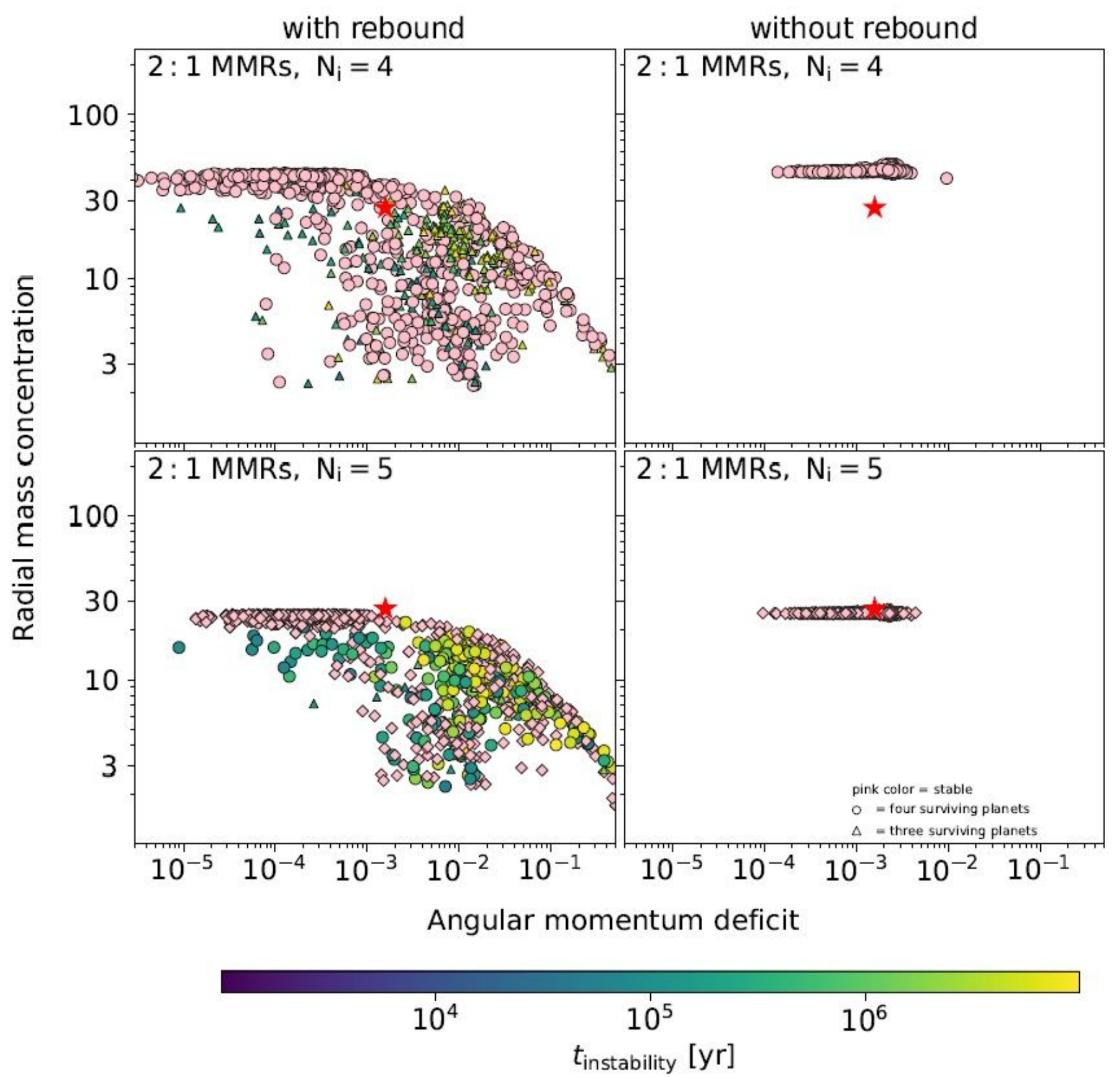

Figure 5

The radial mass concentration and normalized angular momentum deficit distributions among surviving planetary systems in our simulations. (see Manuscript file for full caption) 


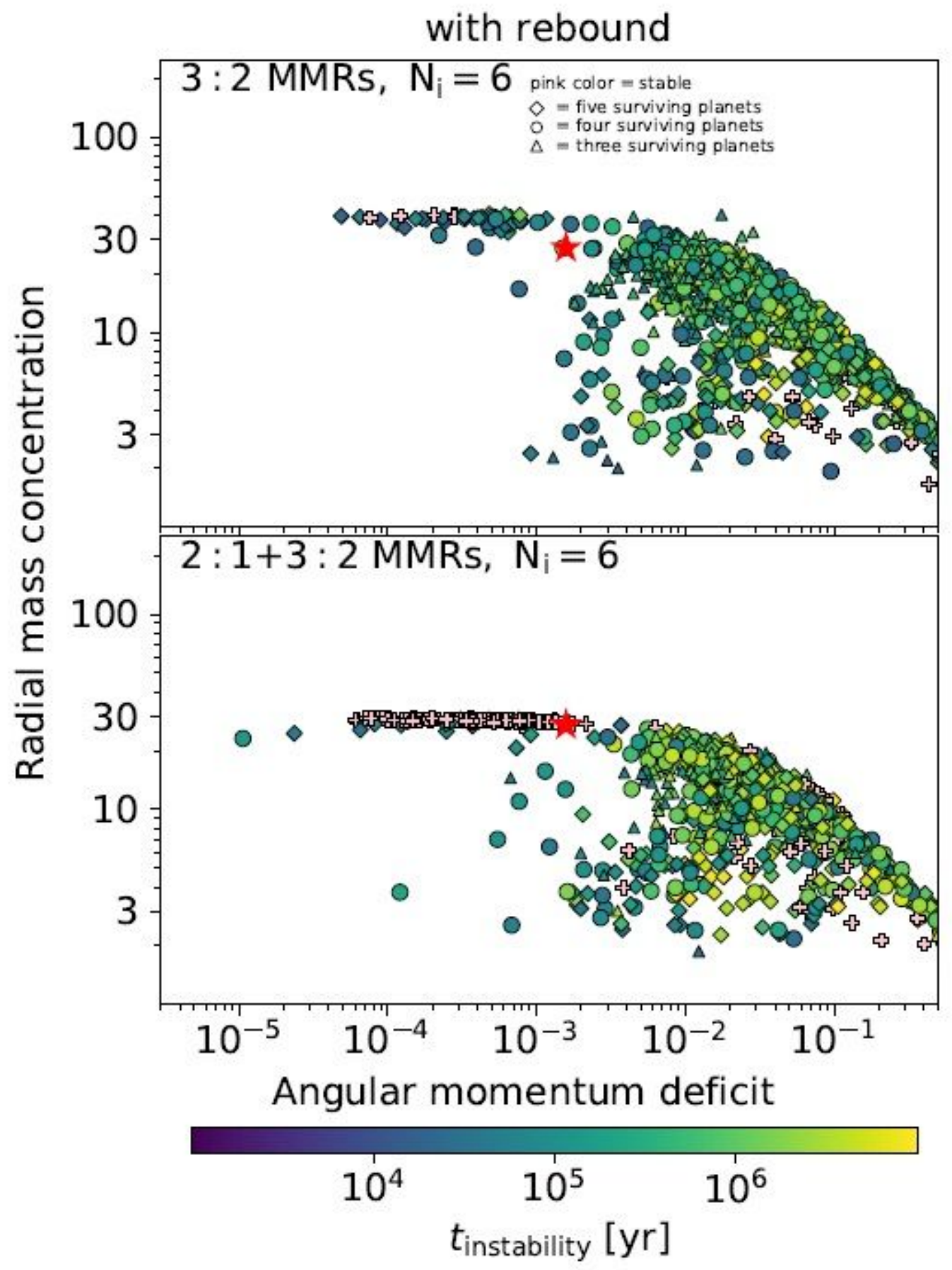

Figure 6

The radial mass concentration and normalized angular momentum deficit distributions among surviving planetary systems in our simulations.(see Manuscript file for full caption) 

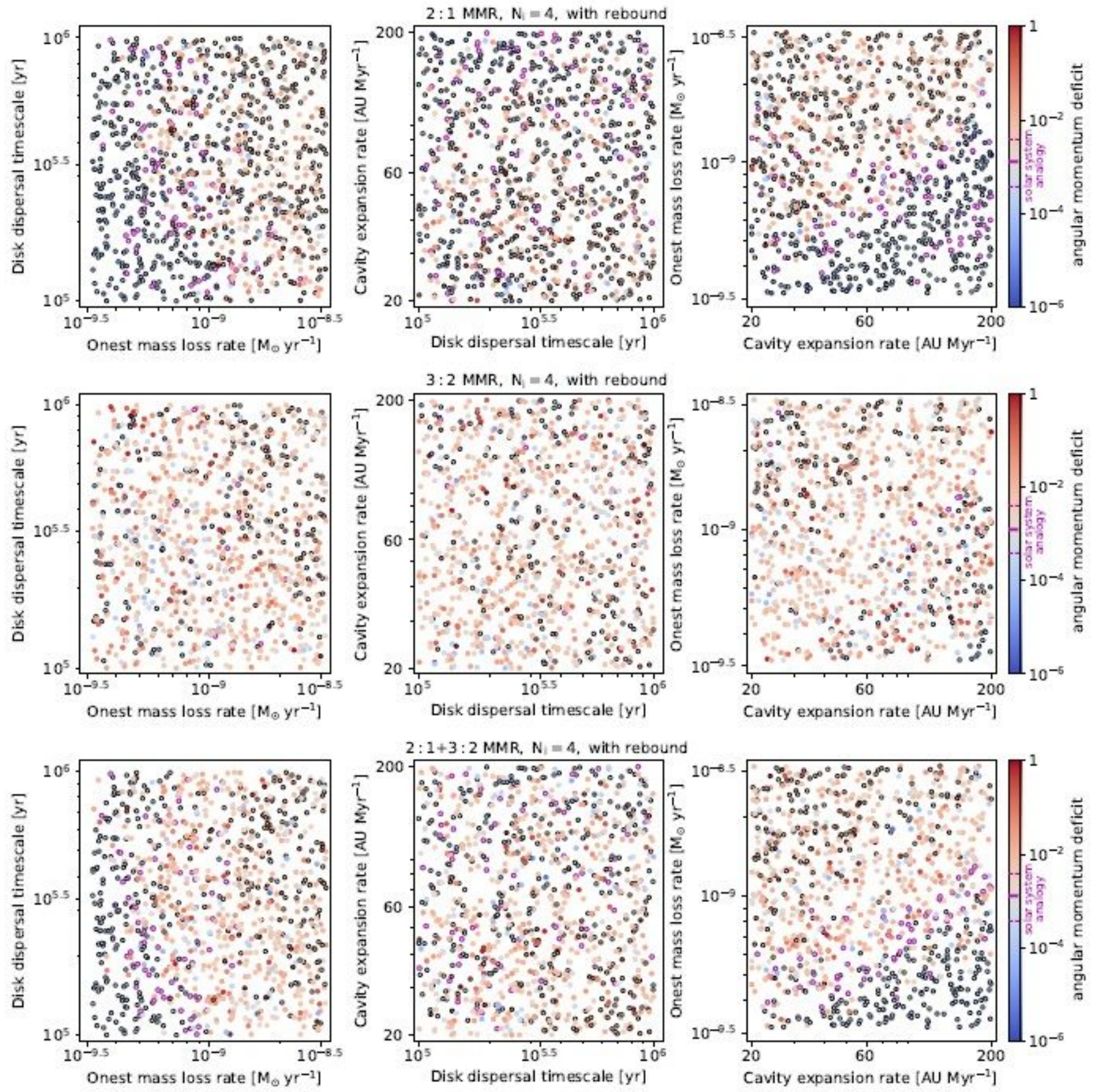

Figure 7

Simulation outcomes of systems with initial four planets as a function of disk parameters. (see Manuscript file for full caption) 

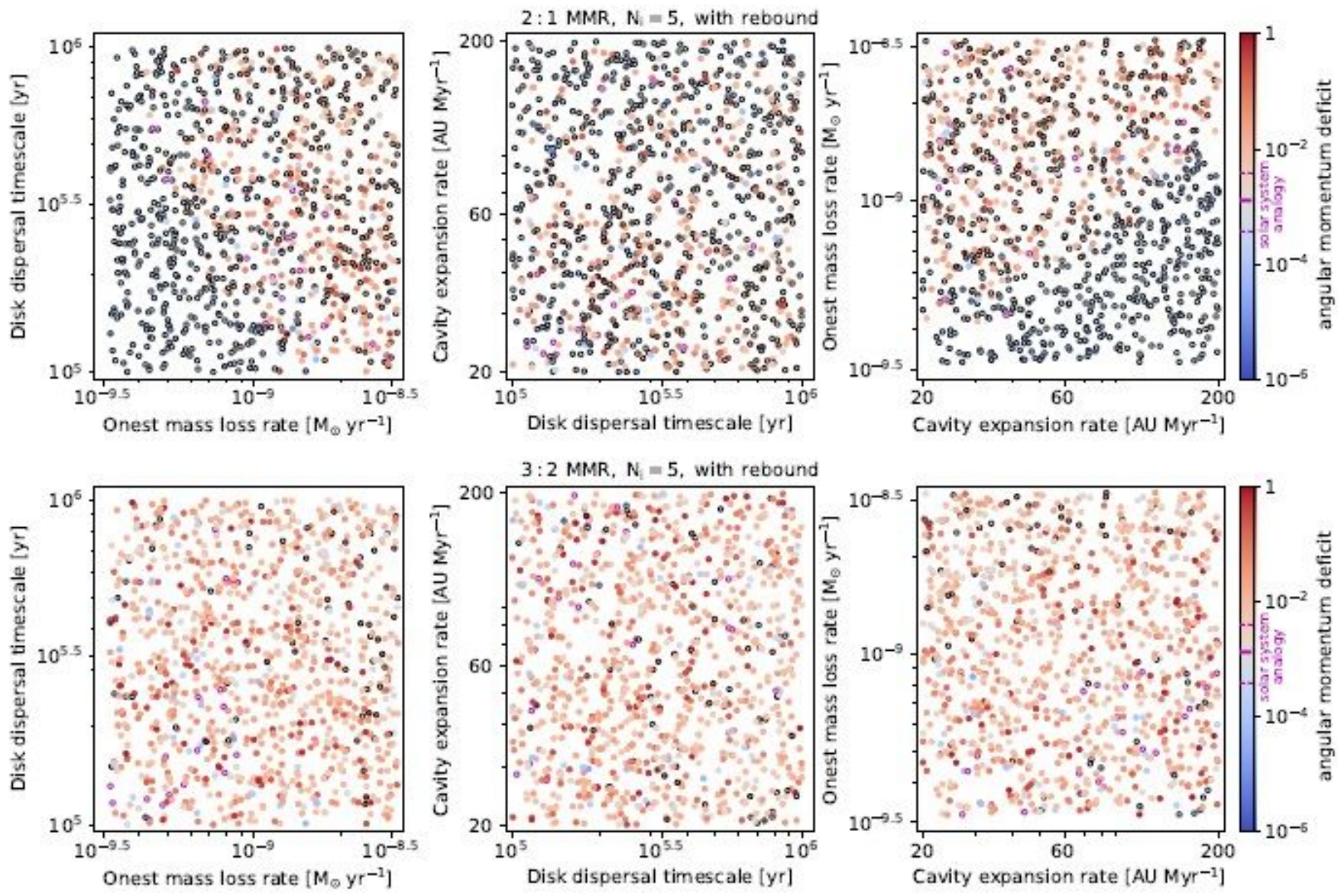

Figure 8

Simulation outcomes of systems with initial five planets as a function of disk parameters.(see Manuscript file for full caption) 

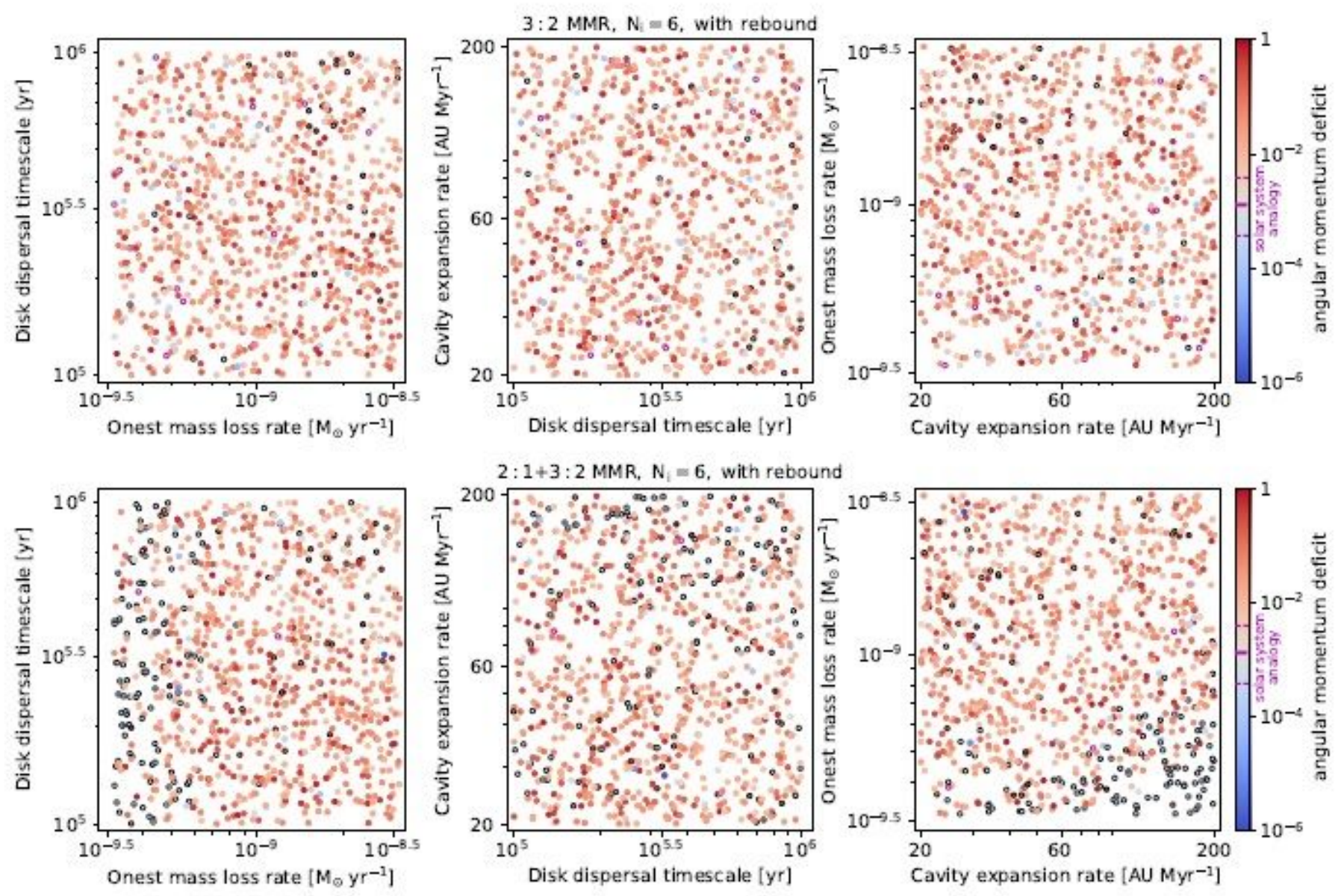

Figure 9

Simulation outcomes of systems with initial six planets as a function of disk parameters. (see Manuscript file for full caption) 